\title{
Intrathecal Therapy for Cancer and Non-Cancer Pain
}

Salim M. Hayek, MD, PhD', Timothy R. Deer, MD², Jason E. Pope, MD³, Sunil J. Panchal, MD, and Vikram Patel, MD ${ }^{5}$

From: ${ }^{1}$ University Hospitals and Outcomes Research Consortium,

Cleveland, $\mathrm{OH} ;{ }^{2}$ Center for

Pain Relief, Charleston, WV;

${ }^{3}$ Napa Pain Institute, Napa,

$\mathrm{CA} ;{ }^{4}$ National Institute of Pain,

Tampa, FL; and ${ }^{5} \mathrm{ACMI}$ Pain Care,

Algonquin, IL.

Dr. Hayek is Associate Professor,

Department of Anesthesiology,

Chief of the Division of Pain

Medicine, University Hospitals

of Cleveland, Cleveland, $\mathrm{OH}$;

and a member of the Outcomes

Research Consortium,

Cleveland, $\mathrm{OH}$. Dr. Deer is

Medical Director, The Center

for Pain Relief, and Clinical

Professor, Anesthesiology, West

Virginia University School of

Medicine, Charleston, WV. Dr.

Pope is partner, Napa Pain

Institute, Napa, CA. Dr. Panchal

is President of the National

Institute of Pain, Tampa, FL.

Dr. Patel is Medical Director of ACMI Pain Care, Algonquin, IL.

Address Correspondence:

Salim M. Hayek, MD, PhD

Division of Pain Medicine University Hospitals 11100 Euclid Ave Cleveland, $\mathrm{OH} 44106$ Email: salim.hayek@ UHhospitals.org

Disclosures and conflict of interest on page ???

Manuscript received Revised manuscript received: Accepted for publication: 04/014/2011

Free full manuscript: www.painphysicianjournal.com
Background: Intrathecal drug infusion therapy is usually considered when spinal-acting analgesics or antispasmodics administered via the oral or transdermal routes fail to control patients' pain or are associated with unacceptable side effects. The intrathecal administration of centrally acting agents bypasses the blood-brain-barrier resulting in much higher cerebrospinal fluid (CSF) concentrations while using reduced amounts of medication to achieve equipotent doses. The intrathecal approach is associated with higher rates of satisfactory pain relief and lower rates of treatment failures and technical complications compared to the epidural route. A paucity of randomized controlled trials (RCTs) has led to concern regarding proper use, selection criteria, and safety of these devices. Cost effectiveness and comparative therapies have now also become a focus of discussion.

Objective: The purpose of this systematic review is to evaluate and update the available evidence for the efficacy and safety of intrathecal infusions used in long-term management (> 6 months) of chronic pain. This paper will not focus on intrathecal administration for spasticity or movement disorders.

Study Design: A systematic review of intrathecal infusion through implanted drug delivery devices for chronic pain.

Methods: Literature search through EMBASE, Medline, Cochrane databases, and systematic reviews as well as peer-reviewed non-indexed journals from 1980 to December 2010. Studies are assessed using the Agency for Healthcare Research and Quality (AHRQ) criteria for observational studies and the Cochrane Musculoskeletal Review Group criteria for randomized trials.

The level of evidence was determined using 5 levels of evidence, ranging from Level I to III with 3 subcategories in Level II, based on the quality of evidence developed by the U.S. Preventive Services Task Force (USPSTF).

Outcome Measures: The primary outcome measure for chronic non-cancer is pain relief (short-term relief $\leq$ one-year and long-term > one-year), whereas it is 3 months for cancer. Secondary outcome measures of improvement in functional status, psychological status, return to work, and reduction in opioid intake.

Results: The level of evidence for this systematic review of non-cancer pain studies meeting the inclusion criteria of continuous use of an intrathecal drug delivery system (IDDS) for at least 12 months duration with at least 25 patients in the cohort, is Level II-3 based on USPSTF criteria. The level of evidence for this systemic review for cancer-related pain studies meeting the inclusion criteria of continuous use of IDDS for at least 3 months duration with at least 25 patients in the cohort is Level $\|-2$ based on USPSTF criteria.

Conclusion: Based on the available evidence, the recommendation for intrathecal infusion systems for cancer-related pain is moderate recommendation based on the high quality of evidence and the recommendation is limited to moderate based on the moderate quality of evidence from nonrandomized studies for non-cancer related pain.

Key words: Intrathecal infusion, intrathecal drug delivery device, intrathecal drug delivery system, intraspinal infusion, programmable infusion systems, spinal infusion, intra-spinal infusion devices, baclofen infusion, intrathecal opioids

Pain Physician 2011; 14:219-248 
linical administration of intrathecal opioids for chronic pain shortly followed detection of Jmu opioid receptors on the dorsal horn of the spinal cord $(1,2)$. The Food and Drug Administration (FDA) approval of Medtronic's intrathecal drug delivery system (IDDS) in 1991 led to a progressive increase in the use of intrathecal analgesics for the relief of chronic pain, both cancer and non-cancer related (3). Inadequate pain relief with conservative medical therapy and/or intolerable side effects to oral/transdermal opioids or failure of interventional and surgical techniques are the main reasons for considering the intrathecal route of drug administration (4-7).

Chronic pain is a major burden and an expensive health problem in industrialized countries $(5,8)$. Chronic pain can be cancer related and non-cancer related with the latter accounting for the vast majority of patients. Pain of spinal origin, and in particular chronic low back pain, is foremost in prevalence and significance among chronic non-cancer pain $(9,10)$. Surgical rates for back pain have increased dramatically in the US over the past 3 decades, in particular for spinal fusion surgeries despite limited reports of efficacy (11). Failed Back Surgery Syndrome (FBSS) or lumbar post-laminectomy syndrome has become a common modern disorder whereby patients suffer with chronic back and/or leg pain following lumbar spinal surgery. FBSS is the most common indication for IDDS placement (12).

Implanted intrathecal infusion systems bypass the blood brain barrier delivering medications directly into the intrathecal space. Currently, only 2 agents are FDA approved for intrathecal use for pain, namely preservative-free morphine and ziconotide $(13,14)$. However, a number of other agents including bupivacaine, clonidine, and fentanyl are commonly used (15). Consensus guidelines regarding intrathecal drug delivery have been published since 2000 (16) with subsequent updates and revisions (17-19). IDDS have become widely used over the past 2 decades to treat pain of neuropathic, nociceptive, and mixed physiological origin in both cancer and non-cancer disease states. This therapy has been reserved as a late stage treatment for those who have responded to oral or transdermal medications, but were not good candidates for the continuation of that therapy because of side effects or lack of persistent efficacy. The use of these therapies can be life changing and can result in significant pain relief, improved quality of life, and reduced side effects, but these accolades do not come without expense or risks $(5,6,20-28)$. However, despite its tout- ed benefits, questions regarding its efficacy and utility remain.

Multiple systematic reviews and health technology assessments have been published without conclusive evidence leading to continued debate of the clinical effectiveness, cost effectiveness, and safety $(5,6,24,29,30)$. In the recent systematic review by Patel et al (24) only 5 observational studies met inclusion criteria, yielding limited evidence. This systematic review of randomized and non-randomized studies for cancer and non-cancer pain is undertaken to assess the benefits, risks, and efficacy of IDDS based on the currently available literature.

\section{Methods}

\section{Search Strategy}

Bibliographic resources such as PubMed, EMBASE, Cochrane Database of Systematic Reviews, and Cochrane Controlled Trials Register, were used to search for English language studies published from 1966 until October 30, 2010 (the date of the last search). To identify the studies the following criteria were used: a) study design: all designs (prospective, retrospective, technical reports, randomized clinical trials); b) study subjects: subjects with chronic pain including cancer pain patients and non-cancer pain patients with or without history of previous spine surgery; c) types of interventions: IDDS implanted and followed for at least 3 months for cancer pain and 12 months for non-cancer pain; d) keywords used to search were intrathecal pump for pain, intrathecal infusion, spinal infusion, morphine pump, intrathecal drug delivery system, spinal pump, intrathecal therapy. Excluded were case reports, technical reports, surveys, animal studies, and in-vitro pump evaluations.

The search was performed by 2 reviewers, in an unblinded standardized manner, and any discrepancies were evaluated by a third reviewer and consensus was reached.

\section{Study Selection}

Search results from all databases were combined and duplicates were removed. Reference lists from retrieved articles were also reviewed for additional relevant studies that were not identified in our search. All articles were triaged for inclusion by the authors for suitability prior to review. Studies were selected for inclusion if the methods clearly indicated that an IDDS was used for management of chronic pain. The 
relevant data on the methodology and outcome measures were collected. The following criteria were used for inclusion in this review: two unblinded reviewers, using standard practice, carried out the study selection; a third reviewer evaluated any discrepancies and all 3 reached a consensus.

\section{Inclusion Criteria}

The studies included in this review had to meet the following criteria: Studies should clearly show the use of intrathecal infusion device/system (programmable or fixed infusion rate) implanted for chronic pain for long-term use. Studies must have a specific indication for intrathecal infusion and the drug injected. A minimum of 3 months of follow-up was available for studies on cancer pain patients. A minimum of 12 months of follow-up was available for studies on non-cancer pain or studies involving both cancer and non-cancer pain patients. Clear documentation of patient outcomes and complications should have been provided. Number of patients evaluated must have been at least 24 .

Inclusion criteria were assessed by 2 unblinded reviewers, in a standardized manner, and a third reviewer evaluated any discrepancies and consensus was reached.

\section{Exclusion Criteria}

Lack of clear documentation of infusion systems or mixed delivery methods. Externalized infusion systems for short-term use. Studies for chronic non-cancer pain with less than 12 months follow-up and studies of cancer related pain with less than 3-month follow-up. Case reports, technical reports, surveys, animal studies, and in-vitro pump evaluations.

Three reviewers were involved: 2 unblinded reviewers, using standard methods, assessed exclusion criteria; the third reviewer became involved if a discrepancy happened and working with the other reviewers, reached a consensus.

\section{Types of Outcome Measures}

\section{Primary Outcome Measures}

Significant pain relief defined as a minimum of 2-point drop on an 11-point numerical pain scale or a decrease of baseline pain intensity by $30 \%$ (31).

\section{Secondary Outcome Measures}

Improvement of function, reduction in the amount of oral medication, decrease in side effects from systemic drugs, and improvement in quality of life (QOL).
Pain and symptom improvement is evaluated on both a short-term (12 months or less) and long-term (more than 12 months) basis for non-cancer pain studies and for 3-months for cancer pain studies.

Working in a standardized manner, outcome measures were assessed by 2 unblinded reviewers, and any discrepancies were evaluated by a third reviewer and consensus was reached.

\section{Review Methods}

\section{Methodological Quality Assessment}

The quality of individual articles was evaluated using criteria from the AHRQ publication (Table 1) for observational studies (32-34) and using Cochrane review criteria for RCTs (Table 2) (35-41) with consensus-based weighted scoring utilized by the guidelines committee of the American Society of Interventional Pain Physicians (ASIPP) and others (24,40-64). Only the studies scoring at least 50 of 100 on weighted scoring criteria were utilized for analysis.

The methodologic quality assessment was performed by 2 reviewers, in an unblinded standardized manner, and any discrepancies were evaluated by a third reviewer and consensus was reached.

\section{Data Extraction}

A standardized form was used to extract the relevant data on the methods used, participants, interventions, outcome measures used and timing of outcome measurement, reported side effects, and the main results. Each study was evaluated by 2 physicians for the stated criteria and any disagreements were resolved by a third physician. If there was a conflict of interest with the reviewed manuscripts with authorship or any other type of conflict, the involved authors did not review the manuscripts for quality assessment, clinical relevance, evidence synthesis, or grading of evidence.

\section{Levels of Evidence}

The levels of evidence in making recommendations for care were adapted from the third USPSTF for the AHRQ criteria. Qualitative analysis was conducted using 5 levels of evidence as described by AHRQ, ranging from Level I to Level III with 3 subcategories in Level II, as illustrated in Table 3 (65). The level of evidence as assessed by 2 reviewers, in an unblinded standardized manner, and any discrepancies were evaluated by a third reviewer and consensus was reached. 
Table 1. Modified AHRQ quality assessment criteria for observational studies.

\begin{tabular}{|c|c|}
\hline CRITERION & Weighted Score (points) \\
\hline 1. Study Question & 2 \\
\hline \multicolumn{2}{|l|}{ - Clearly focused and appropriate question } \\
\hline 2. Study Population & 8 \\
\hline - Description of study population & 5 \\
\hline - Sample size justification & 3 \\
\hline 3. Comparability of Subjects for All Observational Studies & 22 \\
\hline - Specific inclusion/exclusion criteria for all groups & 5 \\
\hline - Criteria applied equally to all groups & 3 \\
\hline - Comparability of groups at baseline with regard to disease status and prognostic factors & 3 \\
\hline - Study groups comparable to non-participants with regard to confounding factors & 3 \\
\hline - Use of concurrent controls & 5 \\
\hline - Comparability of follow-up among groups at each assessment & 3 \\
\hline 4. Exposure or Intervention & 11 \\
\hline - Clear definition of exposure & 5 \\
\hline - Measurement method standard, valid and reliable & 3 \\
\hline - Exposure measured equally in all study groups & 3 \\
\hline 5. Outcome measures & 20 \\
\hline - Primary/secondary outcomes clearly defined & 5 \\
\hline - Outcomes assessed blind to exposure or intervention & 5 \\
\hline - Method of outcome assessment standard, valid and reliable & 5 \\
\hline - Length of follow-up adequate for question & 5 \\
\hline 6. Statistical Analysis & 19 \\
\hline - Statistical tests appropriate & 5 \\
\hline - Multiple comparisons taken into consideration & 3 \\
\hline - Modeling and multivariate techniques appropriate & 2 \\
\hline - Power calculation provided & 2 \\
\hline - Assessment of confounding & 5 \\
\hline - Dose-response assessment if appropriate & 2 \\
\hline 7. Results & 8 \\
\hline - Measure of effect for outcomes and appropriate measure of precision & 5 \\
\hline - Adequacy of follow-up for each study group & 3 \\
\hline 8. Discussion & 5 \\
\hline \multicolumn{2}{|l|}{ - Conclusions supported by results with possible biases and limitations taken into consideration } \\
\hline 9. Funding or Sponsorship & 5 \\
\hline \multicolumn{2}{|l|}{ - Type and sources of support for study } \\
\hline TOTAL SCORE & 100 \\
\hline
\end{tabular}

Adapted and modified from West S et al. Systems to Rate the Strength of Scientific Evidence, Evidence Report, Technology Assessment No. 47. AHRQ Publication No. 02-E016 (172). 
Table 2. Modified and weighted Cochrane methodologic quality assessment criteria as described by Koes et al.

\begin{tabular}{|c|c|c|}
\hline \multicolumn{2}{|c|}{ CRITERION } & $\begin{array}{l}\text { Weighted } \\
\text { Score }\end{array}$ \\
\hline \multicolumn{2}{|c|}{ 1. Study population } & 35 \\
\hline A & Homogeneity & 2 \\
\hline $\mathrm{B}$ & Comparability of relevant baseline characteristics & 5 \\
\hline $\mathrm{C}$ & Randomization procedure adequate & 4 \\
\hline $\mathrm{D}$ & Drop-outs described for each study group separately & 3 \\
\hline \multirow[t]{2}{*}{$\mathrm{E}$} & $<20 \%$ loss for follow-up & 2 \\
\hline & $<10 \%$ loss for follow-up & 2 \\
\hline \multirow[t]{2}{*}{$\mathrm{F}$} & $>50$ subject in the smallest group & 8 \\
\hline & $>100$ subjects in the smallest group & 9 \\
\hline \multicolumn{2}{|c|}{ 2. Interventions } & 25 \\
\hline G & Interventions included in protocol and described & 10 \\
\hline $\mathrm{H}$ & Pragmatic study & 5 \\
\hline I & Co-interventions avoided & 5 \\
\hline $\mathrm{J}$ & Placebo-controlled & 5 \\
\hline \multicolumn{2}{|c|}{ 3. Effect } & 30 \\
\hline K & Patients blinded & 5 \\
\hline $\mathrm{L}$ & Outcome measures relevant & 10 \\
\hline M & Blinded outcome assessments & 10 \\
\hline $\mathrm{N}$ & Follow-up period adequate & 5 \\
\hline \multicolumn{2}{|c|}{ 4. Data-presentation and analysis } & 10 \\
\hline $\mathrm{O}$ & Intention-to-treat analysis & 5 \\
\hline $\mathrm{P}$ & Frequencies of most important outcomes presented for each treatment group & 5 \\
\hline \multicolumn{2}{|c|}{ TOTAL SCORE } & 100 \\
\hline
\end{tabular}

Adapted from Koes BW et al. Efficacy of epidural steroid injections for low-back pain and sciatica: A systematic review of randomized clinical trials. Pain 1995; 63:279-288 (35).

Table 3. Quality of evidence developed by AHRQ.

\begin{tabular}{|l|l|}
\hline I: & Evidence obtained from at least one properly randomized controlled trial. \\
\hline II-1: & Evidence obtained from well-designed controlled trials without randomization. \\
\hline II-2: & $\begin{array}{l}\text { Evidence obtained from well-designed cohort or case-control analytic studies, preferably from more than one center or research } \\
\text { group. }\end{array}$ \\
\hline II-3: & $\begin{array}{l}\text { Evidence obtained from multiple time series with or without the intervention. Dramatic results in uncontrolled experiments (such } \\
\text { as the results of the introduction of penicillin treatment in the 1940s) could also be regarded as this type of evidence. }\end{array}$ \\
\hline III: & Opinions of respected authorities, based on clinical experience descriptive studies and case reports or reports of expert committees. \\
\hline
\end{tabular}

Adapted from the Agency for Healthcare Research and Quality, U.S. Preventive Services Task Force (USPSTF) (65). 
Table 4. Quality of evidence criteria.

\begin{tabular}{|c|c|c|c|}
\hline $\begin{array}{c}\text { Grade of Recommendation/ } \\
\text { Description }\end{array}$ & $\begin{array}{l}\text { Benefit vs Risk and } \\
\text { Burdens }\end{array}$ & $\begin{array}{l}\text { Methodological Quality of } \\
\text { Supporting Evidence }\end{array}$ & Implications \\
\hline $\begin{array}{l}1 \mathrm{~A} / \text { strong recommendation, } \\
\text { high-quality evidence }\end{array}$ & $\begin{array}{l}\text { Benefits clearly outweigh } \\
\text { risk and burdens, or vice } \\
\text { versa }\end{array}$ & $\begin{array}{l}\text { RCTs without important limitations } \\
\text { or overwhelming evidence from } \\
\text { observational studies }\end{array}$ & $\begin{array}{l}\text { Strong recommendation, can apply to } \\
\text { most patients in most circumstances } \\
\text { without reservation }\end{array}$ \\
\hline $\begin{array}{l}1 \mathrm{~B} / \text { strong recommendation, } \\
\text { moderate quality evidence }\end{array}$ & $\begin{array}{l}\text { Benefits clearly outweigh } \\
\text { risk and burdens, or vice } \\
\text { versa }\end{array}$ & $\begin{array}{l}\text { RCTs with important limitations } \\
\text { (inconsistent results, methodologi- } \\
\text { cal flaws, indirect, or imprecise) or } \\
\text { exceptionally strong evidence from } \\
\text { observational studies }\end{array}$ & $\begin{array}{l}\text { Strong recommendation, can apply to } \\
\text { most patients in most circumstances } \\
\text { without reservation }\end{array}$ \\
\hline $\begin{array}{l}\text { 1C/strong recommendation, } \\
\text { low-quality or very low-quality } \\
\text { evidence }\end{array}$ & $\begin{array}{l}\text { Benefits clearly outweigh } \\
\text { risk and burdens, or vice } \\
\text { versa }\end{array}$ & Observational studies or case series & $\begin{array}{l}\text { Strong recommendation but may } \\
\text { change when higher quality evidence } \\
\text { becomes available }\end{array}$ \\
\hline $\begin{array}{l}\text { 2A/weak recommendation, high- } \\
\text { quality evidence }\end{array}$ & $\begin{array}{l}\text { Benefits closely balanced } \\
\text { with risks and burden }\end{array}$ & $\begin{array}{l}\text { RCTs without important limitations } \\
\text { or overwhelming evidence from } \\
\text { observational studies }\end{array}$ & $\begin{array}{l}\text { Weak recommendation, best action } \\
\text { may differ depending on circumstanc- } \\
\text { es or patients' or societal values }\end{array}$ \\
\hline $\begin{array}{l}2 \mathrm{~B} / \text { weak recommendation, } \\
\text { moderate-quality evidence }\end{array}$ & $\begin{array}{l}\text { Benefits closely balanced } \\
\text { with risks and burden }\end{array}$ & $\begin{array}{l}\text { RCTs with important limitations } \\
\text { (inconsistent results, methodologi- } \\
\text { cal flaws, indirect, or imprecise) or } \\
\text { exceptionally strong evidence from } \\
\text { observational studies }\end{array}$ & $\begin{array}{l}\text { Weak recommendation, best action } \\
\text { may differ depending on circumstanc- } \\
\text { es or patients' or societal values }\end{array}$ \\
\hline $\begin{array}{l}\text { 2C/weak recommendation, } \\
\text { low-quality or very low-quality } \\
\text { evidence }\end{array}$ & $\begin{array}{l}\text { Uncertainty in the esti- } \\
\text { mates of benefits, risks, } \\
\text { and burden; benefits, risk, } \\
\text { and burden may be closely } \\
\text { balanced }\end{array}$ & Observational studies or case series & $\begin{array}{l}\text { Very weak recommendations; other } \\
\text { alternatives may be equally reasonable }\end{array}$ \\
\hline
\end{tabular}

Adapted from Guyatt G, et al. Grading strength of recommendations and quality of evidence in clinical guidelines. Report from an American College of Chest Physicians task force. Chest 2006; 129:174-181 (66).

\section{Grading Recommendations}

Qualitative recommendations relative to the quality of evidence for each outcome was judged based on criteria established by Guyatt et al (66) as shown in Table 4. Two reviewers assessed grading recommendations, in an unblinded standardized manner, and a third reviewer evaluated any discrepancies and consensus was reached.

\section{Results}

Figure 1 describes the literature search and review conducted for intrathecal drug delivery systems for non-cancer pain. After comprehensive review and grading based on AHRQ assessment criteria for observational studies, 20 studies were identified that met the specified inclusion and exclusion criteria for intrathecal drug delivery for cancer and non-cancer chronic pain $(20-22,67-83)$. Of these, 15 observational studies involved predominantly non-cancer pain patients (20$22,67-73,75-79)$ and 5 studies examined only cancer pain patients $(74,80-83)$. Of these, 4 were observational cancer pain studies $(74,80-82)$ and one RCT $(83)$ involving only cancer pain patients were identified.

\section{Methodological Quality Assessment}

There were numerous studies on intrathecal drug delivery for cancer pain that were excluded from this literature analysis because of sample size less than 25 patients, lack of 3-month data, or use of externalized pump system (84-90). The follow-up requirement for this review for cancer pain studies was eased down to 3 months compared to 12 months for non-cancer pain; the 3-month mark is more appropriate given the limited lifespan of many cancer pain patients who are considered for intrathecal therapy. Multiple studies for non-cancer pain were reviewed that narrowly failed to meet the inclusion criteria because of sample size $<25$ patients, lack of 12 month follow-up, or the survey nature of the study (91-97). 


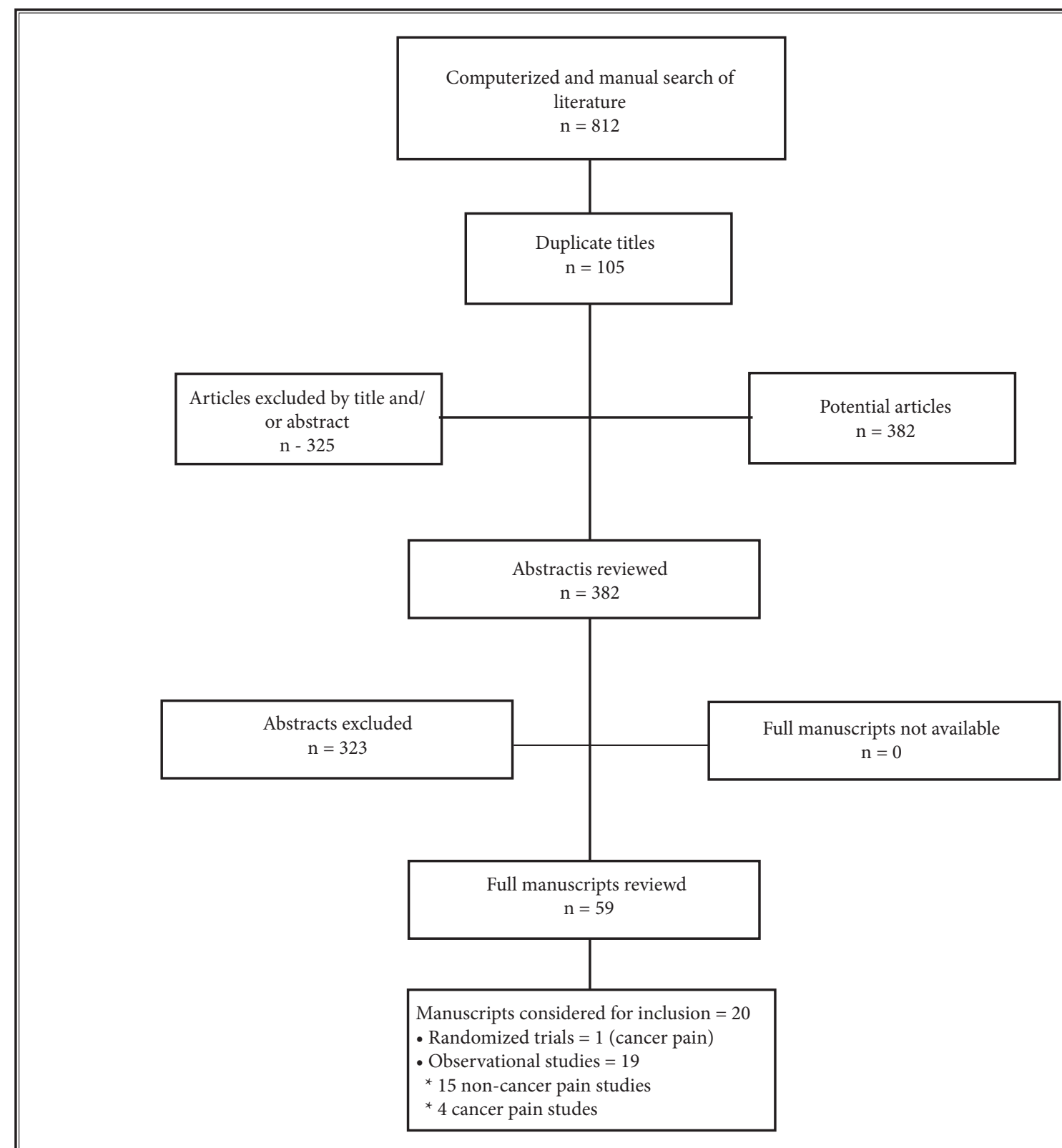

Fig. 1. The flow diagram illustrating published literature evaluating intrathecal infustion systems.

Tables 5, 6, and 7 illustrate the methodological quality assessment of studies. Only studies meeting the aforementioned inclusion and exclusion criteria were included; studies with scores $<50$ were excluded from analysis.

\section{Study Characteristics}

For cancer related pain, 4 observational studies (74,80-82) and one RCT (83) were identified. Methodological quality scores are displayed in Tables 5 and 6 . No RCTs meeting the inclusion criteria were identified 
Table 5. Methodological quality of randomized controlled studies.

\begin{tabular}{|c|c|c|c|}
\hline \multicolumn{2}{|r|}{ CRITERION } & Weighted Score & Smith et al, $2002(83)$ \\
\hline \multicolumn{2}{|c|}{ Study population } & 35 & 26 \\
\hline A & Homogeneity & 2 & 2 \\
\hline B & Comparability of relevant baseline characteristics & 5 & 5 \\
\hline $\mathrm{C}$ & Randomization procedure adequate & 4 & 4 \\
\hline $\mathrm{D}$ & Drop-outs described for each study group separately & 3 & 3 \\
\hline \multirow{2}{*}{$\mathrm{E}$} & $<20 \%$ loss for follow-up & 2 & 2 \\
\hline & $<10 \%$ loss for follow-up & 2 & 2 \\
\hline \multirow{2}{*}{$\mathrm{F}$} & $>50$ subject in the smallest group & 8 & 8 \\
\hline & $>100$ subjects in the smallest group & 9 & - \\
\hline \multicolumn{2}{|l|}{ Interventions } & 25 & 18 \\
\hline G & Interventions included in protocol and described & 10 & 10 \\
\hline $\mathrm{H}$ & Pragmatic study & 5 & 5 \\
\hline I & Co-interventions avoided & 5 & 3 \\
\hline $\mathrm{J}$ & Placebo-controlled & 5 & - \\
\hline \multicolumn{2}{|l|}{ Effect } & 30 & 15 \\
\hline K & Patients blinded & 5 & - \\
\hline $\mathrm{L}$ & Outcome measures relevant & 10 & 10 \\
\hline M & Blinded outcome assessments & 10 & - \\
\hline $\mathrm{N}$ & Follow-up period adequate & 5 & 5 \\
\hline \multicolumn{2}{|r|}{ Data-presentation and analysis } & 10 & 5 \\
\hline $\mathrm{O}$ & Intention-to-treat analysis & 5 & - \\
\hline $\mathrm{P}$ & $\begin{array}{l}\text { Frequencies of most important outcomes presented for } \\
\text { each treatment group }\end{array}$ & 5 & 5 \\
\hline \multicolumn{2}{|r|}{ TOTAL SCORE } & 100 & 64 \\
\hline
\end{tabular}

for non-cancer pain. Fifteen observational studies in non-cancer related pain were identified that met our criteria and included 8 prospective studies and 7 retrospective studies. These studies involved predominantly non-cancer pain patients; a few studies had a minority of patients with cancer-related pain $(21,68,69,72)$. Table 7 describes scoring of non-cancer pain studies analyzed via the methodological assessment for intrathecal drug delivery in patients with primarily non-cancer chronic pain in reverse chronological order as described in Methods.

Descriptive criteria of eligible cancer and noncancer pain studies are displayed in Tables 8 and 9, respectively. All studies used pain scores, visual analogue scale (VAS) or numeric rating scale (NRS) as outcome measures. Additional outcome measures included Mc-
Gill Pain Questionnaires (MPQ) $(20,67,70,78,79)$, medications used $(21,22,68,69,73,75,81-83)$, drug toxicity (83), functional outcomes including Oswestry scores in back pain patients $(22,70,78)$, works status in some non-cancer pain studies $(20,22,70,75)$ as well as other functional parameters specific to the study or condition $(21,70,72,76,81-83)$ or pump parameters (77). All but 3 non-cancer studies examined complications $(20,70,77)$. All studies (cancer and non-cancer related pain) reported positive outcomes. All studies examining pain relief reported improvements in pain scores in patients receiving intrathecal analgesic therapy (20-22,67-70,72-76,78-83).

Study particulars for some of the non-cancer pain studies $(69,75,76,78,79)$ were detailed in the systematic review by Patel et al (24). A recent study by Duse et al 
Intrathecal Therapies for Cancer and Non-Cancer Pain

Table 6. Methodological quality of observational studies for cancer related pain.

\begin{tabular}{|c|c|c|c|c|c|}
\hline CRITERION & $\begin{array}{c}\text { Weighted } \\
\text { Score }\end{array}$ & $\begin{array}{c}\text { Rauck et al, } \\
2003(74)\end{array}$ & $\begin{array}{c}\text { Becker et al, } \\
2000(80)\end{array}$ & $\begin{array}{c}\text { Onofrio and } \\
\text { Yaksh, } 1990 \\
(82)\end{array}$ & $\begin{array}{c}\text { Penn and } \\
\text { Paice, } 1987 \\
\text { (81) }\end{array}$ \\
\hline 1. Study Question & 2 & 2 & 2 & 2 & 2 \\
\hline - Clearly focused and appropriate question & 2 & 2 & 2 & 2 & 2 \\
\hline 2. Study Population & 8 & 5 & 5 & 5 & 5 \\
\hline - Description of study population & 5 & 5 & 5 & 5 & 5 \\
\hline - Sample size justification & 3 & - & - & - & - \\
\hline 3. Comparability of Subjects for All Observational Studies & 22 & 9 & 9 & 11 & 10 \\
\hline - Specific inclusion/exclusion criteria for all groups & 5 & 4 & 3 & 4 & 3 \\
\hline - Criteria applied equally to all groups & 3 & 1 & 3 & 3 & 3 \\
\hline $\begin{array}{l}\text { - Comparability of groups at baseline with regard to disease status and } \\
\text { prognostic factors }\end{array}$ & 3 & 1 & 1 & 3 & 1 \\
\hline $\begin{array}{l}\text { - Study groups comparable to non-participants with regard to confounding } \\
\text { factors }\end{array}$ & 3 & - & - & - & - \\
\hline - Use of concurrent controls & 5 & - & - & - & - \\
\hline - Comparability of follow-up among groups at each assessment & 3 & 3 & 2 & 1 & 3 \\
\hline 4. Exposure or Intervention & 11 & 11 & 11 & 7 & 8 \\
\hline - Clear definition of exposure & 5 & 5 & 5 & 3 & 5 \\
\hline - Measurement method standard, valid and reliable & 3 & 3 & 3 & 3 & 3 \\
\hline - Exposure measured equally in all study groups & 3 & 3 & 3 & 1 & - \\
\hline 5. Outcome measures & 20 & 14 & 10 & 13 & 12 \\
\hline - Primary/secondary outcomes clearly defined & 5 & 5 & 2 & 4 & 4 \\
\hline - Outcomes assessed blind to exposure or intervention & 5 & - & - & - & - \\
\hline - Method of outcome assessment standard, valid and reliable & 5 & 4 & 4 & 4 & 3 \\
\hline - Length of follow-up adequate for question & 5 & 5 & 4 & 5 & 5 \\
\hline 6. Statistical Analysis & 19 & 8 & 5 & 12 & 6 \\
\hline - Statistical tests appropriate & 5 & 5 & 2 & 5 & 5 \\
\hline - Multiple comparisons taken into consideration & 3 & 3 & 3 & 3 & - \\
\hline - Modeling and multivariate techniques appropriate & 2 & - & - & 2 & - \\
\hline - Power calculation provided & 2 & - & - & - & - \\
\hline - Assessment of confounding & 5 & - & - & 1 & - \\
\hline - Dose-response assessment if appropriate & 2 & - & - & 1 & 1 \\
\hline 7. Results & 8 & 6 & 7 & 7 & 7 \\
\hline - Measure of effect for outcomes and appropriate measure of precision & 5 & 5 & 4 & 5 & 4 \\
\hline - Adequacy of follow-up for each study group & 3 & 1 & 3 & 2 & 3 \\
\hline 8. Discussion & 5 & 5 & 5 & 5 & 5 \\
\hline $\begin{array}{l}\text { - Conclusions supported by results with possible biases and limitations taken } \\
\text { into consideration }\end{array}$ & & 5 & 5 & 5 & 5 \\
\hline 9. Funding or Sponsorship & 5 & - & - & 5 & 5 \\
\hline - Type and sources of support for study & & - & - & 5 & 5 \\
\hline TOTAL SCORE & 100 & 60 & 53 & 67 & 60 \\
\hline
\end{tabular}


Pain Physician: May/June 2011; 14:219-248

Table 7. Methodological quality of observational studies for chronic non-cancer pain.

\begin{tabular}{|c|c|c|c|c|c|c|c|c|c|}
\hline CRITERION & $\begin{array}{l}\text { Weighted } \\
\text { Score }\end{array}$ & $\begin{array}{l}\text { Atli et } \\
\text { al } 2010 \\
(68) \\
\end{array}$ & $\begin{array}{l}\text { Duse et } \\
\text { al, 2009 } \\
(20)\end{array}$ & $\begin{array}{l}\text { Ellis et } \\
\mathrm{al}, 2008 \\
(72)\end{array}$ & \begin{tabular}{|l} 
Ilias et \\
al, 2008 \\
$(21)$ \\
\end{tabular} & $\begin{array}{l}\text { Shaladi et } \\
\text { al, 2007 } \\
(76)\end{array}$ & $\begin{array}{l}\text { Staats et } \\
\text { al, 2007 } \\
(77) \\
\end{array}$ & $\begin{array}{l}\text { Doleys et } \\
\text { al } 2006 \\
\text { (70) }\end{array}$ & $\begin{array}{l}\text { Thimineur } \\
\text { et al, } 2004 \\
\text { (78) }\end{array}$ \\
\hline 1. Study Question & 2 & 2 & 2 & 2 & 1 & 1 & 2 & 2 & 2 \\
\hline - Clearly focused and appropriate question & 2 & 2 & 2 & 2 & 1 & 1 & 2 & 2 & 2 \\
\hline 2. Study Population & 8 & 5 & 5 & 5 & 5 & 5 & 5 & 5 & 5 \\
\hline - Description of study population & 5 & 5 & 5 & 5 & 5 & 5 & 5 & 5 & 5 \\
\hline - Sample size justification & 3 & - & - & - & - & - & - & - & - \\
\hline $\begin{array}{l}\text { 3. Comparability of Subjects for All } \\
\text { Observational Studies }\end{array}$ & 22 & 6 & 8 & 13 & 10 & 13 & 9 & 11 & 17 \\
\hline - Specific inclusion/exclusion criteria for all groups & 5 & 1 & 3 & 3 & 3 & 4 & 3 & 4 & 5 \\
\hline - Criteria applied equally to all groups & 3 & 1 & 3 & 1 & 3 & 3 & 3 & 3 & 3 \\
\hline $\begin{array}{l}\text { Comparability of groups at baseline with regard } \\
\text { to disease status and prognostic factors }\end{array}$ & 3 & 1 & 2 & 3 & 1 & 3 & 3 & 1 & 3 \\
\hline $\begin{array}{l}\text { - Study groups comparable to non-participants } \\
\text { with regard to confounding factors }\end{array}$ & 3 & - & - & 3 & - & - & - & - & 3 \\
\hline - Use of concurrent controls & 5 & - & - & - & - & - & - & - & - \\
\hline $\begin{array}{l}\text { - Comparability of follow-up among groups at } \\
\text { each assessment }\end{array}$ & 3 & 3 & - & 3 & 3 & 3 & - & 3 & 3 \\
\hline 4. Exposure or Intervention & 11 & 11 & 11 & 11 & 11 & 11 & 11 & 11 & 6 \\
\hline - Clear definition of exposure & 5 & 5 & 5 & 5 & 5 & 5 & 5 & 5 & 3 \\
\hline $\begin{array}{l}\text { - Measurement method standard, valid and } \\
\text { reliable }\end{array}$ & 3 & 3 & 3 & 3 & 3 & 3 & 3 & 3 & 3 \\
\hline - Exposure measured equally in all study groups & 3 & 3 & 3 & 3 & 3 & 3 & 3 & 3 & - \\
\hline 5. Outcome measures & 20 & 15 & 15 & 15 & 12 & 15 & 15 & 15 & 13 \\
\hline - Primary/secondary outcomes clearly defined & 5 & 5 & 5 & 5 & 2 & 5 & 5 & 5 & 4 \\
\hline $\begin{array}{l}\text { Outcomes assessed blind to exposure or } \\
\text { intervention }\end{array}$ & 5 & - & - & - & - & - & - & - & - \\
\hline $\begin{array}{l}\text { - Method of outcome assessment standard, valid } \\
\text { and reliable }\end{array}$ & 5 & 5 & 5 & 5 & 5 & 5 & 5 & 5 & 4 \\
\hline - Length of follow-up adequate for question & 5 & 5 & 5 & 5 & 5 & 5 & 5 & 5 & 5 \\
\hline 6. Statistical Analysis & 19 & 12 & 8 & 15 & 8 & 10 & 8 & 11 & 4 \\
\hline - Statistical tests appropriate & 5 & 5 & 5 & 5 & 5 & 5 & 5 & 5 & 4 \\
\hline - Multiple comparisons taken into consideration & 3 & 3 & 3 & 3 & 3 & 3 & 3 & 3 & - \\
\hline $\begin{array}{l}\text { - Modeling and multivariate techniques } \\
\text { appropriate }\end{array}$ & 2 & 2 & - & 2 & - & 2 & - & - & - \\
\hline - Power calculation provided & 2 & - & - & - & - & - & - & - & - \\
\hline - Assessment of confounding & 5 & - & - & 5 & - & - & - & 3 & - \\
\hline - Dose-response assessment if appropriate & 2 & 2 & - & - & - & - & - & - & - \\
\hline 7. Results & 8 & 8 & 8 & 8 & 8 & 8 & 8 & 8 & 7 \\
\hline $\begin{array}{l}\text { - Measure of effect for outcomes and appropriate } \\
\text { measure of precision }\end{array}$ & 5 & 5 & 5 & 5 & 5 & 5 & 5 & 5 & 4 \\
\hline - Adequacy of follow-up for each study group & 3 & 3 & 3 & 3 & 3 & 3 & 3 & 3 & 3 \\
\hline 8. Discussion & 5 & 5 & 5 & 5 & 5 & 5 & 5 & 5 & 4 \\
\hline $\begin{array}{l}\text { - Conclusions supported by results with possible } \\
\text { biases and limitations taken into consideration }\end{array}$ & & 5 & 5 & 5 & 5 & 5 & 5 & 5 & 4 \\
\hline 9. Funding or Sponsorship & 5 & - & 4 & 4 & 4 & - & 4 & - & 2 \\
\hline - Type and sources of support for study & & - & 4 & 4 & 4 & - & 4 & - & 2 \\
\hline TOTAL SCORE & 100 & 64 & 66 & 78 & 64 & 63 & 67 & 67 & 60 \\
\hline
\end{tabular}


Intrathecal Therapies for Cancer and Non-Cancer Pain

Table 7 cont.. Methodological quality of observational studies for chronic non-cancer pain.

\begin{tabular}{|c|c|c|c|c|c|c|c|c|}
\hline CRITERION & $\begin{array}{l}\text { Weighted } \\
\text { Score }\end{array}$ & $\begin{array}{l}\text { Deer et } \\
\text { al, 2004 } \\
(22)\end{array}$ & $\begin{array}{l}\text { Deer et } \\
\text { al, 2002 } \\
(69)\end{array}$ & $\begin{array}{l}\text { Dominguez } \\
\text { et al, } 2002 \\
\text { (71) }\end{array}$ & $\begin{array}{l}\text { Rainov et } \\
\text { al, 2001 } \\
\text { (73) }\end{array}$ & $\begin{array}{l}\text { Roberts } \\
\text { et al, } 2001 \\
\text { (75) }\end{array}$ & $\begin{array}{l}\text { Anderson } \\
\text { and } \\
\text { Burchiel, } \\
1999(67)\end{array}$ & $\begin{array}{l}\text { Winkelmüller } \\
\& \\
\text { Winkelmüller } \\
1996(79)\end{array}$ \\
\hline 1. Study Question & 2 & - & 2 & 2 & 2 & 2 & 2 & 2 \\
\hline - Clearly focused and appropriate question & 2 & - & 2 & 2 & 2 & 2 & 2 & 2 \\
\hline 2. Study Population & 8 & 5 & 5 & 5 & 5 & 5 & 5 & 4 \\
\hline - Description of study population & 5 & 5 & 5 & 5 & 5 & 5 & 5 & 4 \\
\hline - Sample size justification & 3 & - & - & - & - & - & - & - \\
\hline $\begin{array}{l}\text { 3. Comparability of Subjects for All Observational } \\
\text { Studies }\end{array}$ & 22 & 11 & 5 & 12 & 5 & 3 & 9 & 3 \\
\hline - Specific inclusion/exclusion criteria for all groups & 5 & 2 & 5 & 5 & 5 & 3 & 3 & 3 \\
\hline - Criteria applied equally to all groups & 3 & 2 & - & 3 & - & - & 3 & - \\
\hline $\begin{array}{l}\text { - Comparability of groups at baseline with regard to } \\
\text { disease status and prognostic factors }\end{array}$ & 3 & 2 & - & 1 & - & - & - & - \\
\hline $\begin{array}{l}\text { - Study groups comparable to non-participants with } \\
\text { regard to confounding factors }\end{array}$ & 3 & 2 & - & - & - & - & - & - \\
\hline - Use of concurrent controls & 5 & - & - & - & - & - & - & - \\
\hline $\begin{array}{l}\text { - Comparability of follow-up among groups at each } \\
\text { assessment }\end{array}$ & 3 & 3 & - & 3 & - & - & 3 & - \\
\hline 4. Exposure or Intervention & 11 & 11 & 8 & 11 & 8 & 8 & 8 & 8 \\
\hline - Clear definition of exposure & 5 & 5 & 5 & 5 & 5 & 5 & 5 & 5 \\
\hline - Measurement method standard, valid and reliable & 3 & 3 & 3 & 3 & 3 & 3 & 3 & 3 \\
\hline - Exposure measured equally in all study groups & 3 & 3 & - & 3 & - & - & - & - \\
\hline 5. Outcome measures & 20 & 14 & 13 & 15 & 12 & 12 & 15 & 14 \\
\hline - Primary/secondary outcomes clearly defined & 5 & 5 & 5 & 5 & 4 & 4 & 5 & 4 \\
\hline $\begin{array}{l}\text { - Outcomes assessed blind to exposure or } \\
\text { intervention }\end{array}$ & 5 & - & - & - & - & - & - & - \\
\hline $\begin{array}{l}\text { - Method of outcome assessment standard, valid } \\
\text { and reliable }\end{array}$ & 5 & 4 & 3 & 5 & 3 & 3 & 5 & 5 \\
\hline - Length of follow-up adequate for question & 5 & 5 & 5 & 5 & 5 & 5 & 5 & 5 \\
\hline 6. Statistical Analysis & 19 & 11 & 4 & 4 & 5 & 5 & 8 & 5 \\
\hline - Statistical tests appropriate & 5 & 5 & 4 & 11 & 5 & 5 & 5 & - \\
\hline - Multiple comparisons taken into consideration & 3 & 3 & - & 3 & - & - & 3 & - \\
\hline - Modeling and multivariate techniques appropriate & 2 & - & - & 2 & - & - & - & - \\
\hline - Power calculation provided & 2 & - & - & - & - & - & - & - \\
\hline - Assessment of confounding & 5 & 3 & - & - & - & - & - & - \\
\hline - Dose-response assessment if appropriate & 2 & - & - & 2 & - & - & - & - \\
\hline 7. Results & 8 & 8 & 7 & 8 & 6 & 7 & 8 & 8 \\
\hline $\begin{array}{l}\text { - Measure of effect for outcomes and appropriate } \\
\text { measure of precision }\end{array}$ & 5 & 5 & 4 & 5 & 3 & 4 & 5 & 5 \\
\hline - Adequacy of follow-up for each study group & 3 & 3 & 3 & 3 & 3 & 3 & 3 & 3 \\
\hline 8. Discussion & 5 & 5 & 4 & 5 & 5 & 4 & 5 & 4 \\
\hline $\begin{array}{l}\text { - Conclusions supported by results with possible } \\
\text { biases and limitations taken into consideration }\end{array}$ & & 5 & 4 & 5 & 5 & 4 & 5 & 5 \\
\hline 9. Funding or Sponsorship & 5 & - & 5 & - & 2 & 4 & - & 5 \\
\hline - Type and sources of support for study & & - & 5 & - & 2 & 4 & - & 5 \\
\hline TOTAL SCORE & 100 & 65 & 53 & 62 & 50 & 50 & 60 & 53 \\
\hline
\end{tabular}


Pain Physician: May/June 2011; 14:219-248

Table 8. Summary descriptions of studies for intrathecal drug delivery for cancer-related pain.

\begin{tabular}{|c|c|c|c|c|c|c|}
\hline $\begin{array}{c}\text { Study / } \\
\text { Methods }\end{array}$ & Participants & Intervention & Outcome & Results & $\begin{array}{c}\text { Conclusion(s) } \\
\text { Short-term }<1 \\
\text { year } \\
\end{array}$ & Complications \\
\hline $\begin{array}{l}\text { Rauck et al, } \\
2003 \text { (74) }\end{array}$ & $\begin{array}{l}149 \text { patients were } \\
\text { enrolled, } 119 \\
\text { implanted for } \\
\text { refractory cancer } \\
\text { pain (analgesic doses } \\
\text { caused intolerable } \\
\text { side effects) with } \\
\text { implanted ITP } \\
\text { for morphine } \\
\text { therapy-trialed with } \\
\text { percutaneous bolus } \\
\text { injection with at least } \\
50 \% \text { improvement in } \\
\text { pain, device activated } \\
\text { at } 14 \text { days post } \\
\text { implant }\end{array}$ & $\begin{array}{l}\text { Implantable drug } \\
\text { infusion system } \\
\text { with morphine; self } \\
\text { titration of systemic } \\
\text { opioids }\end{array}$ & $\begin{array}{l}\text { Outcomes included } \\
\text { supplemental } \\
\text { opioid use, opioid } \\
\text { complications, pain } \\
\text { relief, success was } \\
\text { defined as } 50 \% \text { or }> \\
\text { reduction in one of } \\
\text { the aforementioned } \\
3 \text { and global } \\
\text { assessment }\end{array}$ & $\begin{array}{l}\text { NAS decreased } 31 \% \text { and } \\
\text { was maintained through } \\
\text { months } 10-13 \text { in } 15 \text { patients, } \\
\text { systemic opioid use at } 13 \\
\text { months was } 0 \% \text {; opioid } \\
\text { complication data not } \\
\text { available for } 13 \text { months, } \\
\text { rating was excellent in } 87 \% \\
\text { of patients at } 13 \text { months }\end{array}$ & $\begin{array}{l}\text { IT morphine } \\
\text { can decrease } \\
\text { opioid side } \\
\text { effects, systemic } \\
\text { medications, and } \\
\text { improve VAS and } \\
\text { patient satisfaction } \\
\text { at one year in } \\
\text { patients with } \\
\text { refractory cancer } \\
\text { pain }\end{array}$ & $\begin{array}{l}\text { Of the } 119 \text { implants, } \\
7 \text { patients had } \\
\text { device related } \\
\text { malfunctions, } 36 \text { had } \\
\text { procedure related } \\
\text { complications, one } \\
\text { had event related to } \\
\text { intercurrent illness } \\
\text { or injury ( } 40 \text { patients } \\
\text { total) } \\
\text { Of the } 119 \text { cancer } \\
\text { pain patients } \\
\text { implanted, } 15 \text { made } \\
\text { it to } 13 \text { months }\end{array}$ \\
\hline $\begin{array}{l}\text { Smith et al, } \\
2002 \text { (83) }\end{array}$ & $\begin{array}{l}202 \text { patients enrolled } \\
\text { with refractory cancer } \\
\text { pain, multicenter trial } \\
\text { with initial VAS }>\text { or } \\
=5 \text { despite } 200 \mathrm{mg} / \mathrm{d} \\
\text { oral morphine } \\
\text { equivalent, age }>18, \\
\text { life expectancy }>3 \\
\text { months; most had } \\
\text { mixed neuropathic } \\
\text { and nociceptive pain } \\
\text { (60\%); baseline VAS } \\
\text { approximately } 7.5\end{array}$ & $\begin{array}{l}\text { Implanted IDDS to } \\
\text { manage refractory } \\
\text { cancer pain as } \\
\text { compared to CMM }\end{array}$ & $\begin{array}{l}\text { Prospective } \\
\text { multicenter } \\
\text { randomized study, } \\
\text { data collected } \\
\text { baseline } 2,4,6 \text {, } \\
8,10,12 \text { weeks; } \\
\text { primary evaluation } \\
\text { was } 4 \text { weeks after } \\
\text { randomization } \\
\text { comparing CMM } \\
\text { with IDDS; } \\
\text { measured VAS, } \\
\text { toxicity, secondary } \\
\text { outcomes: } \\
\text { individual drug } \\
\text { toxicities, quality } \\
\text { of life, health care } \\
\text { resource, mortality, } \\
\text { opioid dose }\end{array}$ & $\begin{array}{l}\text { 60/71 IDDS patients } \\
\text { achieved success, as } \\
\text { compared to CMM } 51 / 72 \\
\text { patients, IDDS patients had } \\
\text { decreased VAS of at least } 2 \\
\text { points and toxicity ( } 41 / 71 \text { ) } \\
\text { vs } 27 / 72 \text { with CMM; IDDS } \\
\text { decreased } 52 \% \text {, as compared } \\
\text { to } 39 \% \text { reduction, further } \\
\text { IDDS survivability was } \\
\text { better than CMM ( } 53.9 \text { vs } \\
37.2 \text { ) }\end{array}$ & $\begin{array}{l}\text { IDDS improved } \\
\text { pain control, } \\
\text { reduced toxicity } \\
\text { and improved } \\
\text { survival in patients } \\
\text { with refractory } \\
\text { cancer pain. }\end{array}$ & $\begin{array}{l}194 \text { serious adverse } \\
\text { events reported, } \\
\text { split evenly among } \\
\text { the } 2 \text { groups (CMM } \\
95 \text { IDDS 99). } 16 \\
\text { were associated } \\
\text { with implanted } \\
\text { pump or "related } \\
\text { procedure," of these } \\
6 \text { were related to the } \\
\text { pump, } 5 \text { to lumbar } \\
\text { insertion site, and } \\
5 \text { to the catheter; } \\
\text { surgical revision } \\
\text { required in 10, } \\
\text { explanted in one } \\
\text { because of infection }\end{array}$ \\
\hline $\begin{array}{l}\text { Becker et al, } \\
2000(80)\end{array}$ & $\begin{array}{l}43 \text { consecutively } \\
\text { treated cancer } \\
\text { patients, } 19 \text { female } \\
\text { and } 24 \text { male, with a } \\
\text { mean age of } 64 \text { years } \\
\text { (range: } 40-84 \text { years) }\end{array}$ & $\begin{array}{l}\text { In only } 19 \text { patients } \\
\text { was a screening trial } \\
\text { performed mostly } \\
\text { through an epidural } \\
\text { catheter }\end{array}$ & $\begin{array}{l}\text { VRS, best pain } \\
\text { reduction, final } \\
\text { pain reduction }\end{array}$ & $\begin{array}{l}\text { Neuropathic pain reduction } \\
\text { (VRS was } 61.1 \% \text {, nociceptive } \\
\text { pain was } 77.8 \% \text { longer } \\
\text { duration of nociceptive } \\
\text { pain reduction. No more } \\
\text { than moderate pain } \\
\text { reduction was recorded in } \\
\text { patients with peripheral } \\
\text { nerve involvement, nervus } \\
\text { plexus avulsions, and spinal } \\
\text { cord compression did } \\
\text { not show any long-term } \\
\text { benefit of intrathecal opioid } \\
\text { application }\end{array}$ & $\begin{array}{l}\text { IT morphine } \\
\text { is an effective } \\
\text { treatment for } \\
\text { neuropathic and } \\
\text { nociceptive cancer } \\
\text { pain. Best results } \\
\text { were observed for } \\
\text { nociceptive in the } \\
\text { "trunk area of the } \\
\text { body." }\end{array}$ & $\begin{array}{l}\text { Complications from } \\
\text { pump in } 5 \text { patients: } \\
3 \text { spinal catheter } \\
\text { malfunction, pump } \\
\text { pouch hematoma, } \\
\text { post-operative } \\
\text { pneumonia. No } \\
\text { malfunctions of } \\
\text { hardware or local } \\
\text { infection was } \\
\text { reported. }\end{array}$ \\
\hline $\begin{array}{l}\text { Onofrio and } \\
\text { Yaksh } 1990 \\
(82)\end{array}$ & $\begin{array}{l}53 \text { patients with } \\
\text { terminal metastatic } \\
\text { disease; } 24 \text { males, } 29 \\
\text { females, mean age } 58 \\
\text { years, median age } 62 \\
\text { years }\end{array}$ & $\begin{array}{l}\text { Trialed with single } \\
\text { shot morphine } \\
\text { IT trial } 0.5 \text { to } 2 \\
\text { mg) and IDDS } \\
\text { with morphine for } \\
\text { terminal cancer pain, } \\
\text { analgesic effects, } \\
\text { prognostic value } \\
\text { of trial to infusion } \\
\text { success }\end{array}$ & $\begin{array}{l}\text { Parenteral narcotic } \\
\text { use, analgesic index, } \\
\text { mobility, overall } \\
\text { outcome }\end{array}$ & $\begin{array}{l}\text { Analgesic index improved, } \\
\text { mobility improved, } 26 \\
\text { patients had therapy for at } \\
\text { least } 16 \text { weeks, week } 16 \text { IT } \\
\text { infusion was } 9.5 \pm 2.1 \mathrm{mg} / \mathrm{d} \text {, } \\
\text { compared to } 3.7 \pm 0.3 \mathrm{mg} / \mathrm{d} \text {, } \\
65 \% \text { self-reported good or } \\
\text { excellent results }\end{array}$ & $\begin{array}{l}\text { Long-term efficacy } \\
\text { and safety of spinal } \\
\text { opioid infusion } \\
\text { in patients with } \\
\text { terminal cancer is } \\
\text { justified. }\end{array}$ & $\begin{array}{l}\text { Miscalculation of } \\
\text { pump refill dates } \\
\text { leading to severe } \\
\text { pain in } 5 \text { cases, } \\
\text { in one autopsy of } \\
\text { patient with dose } \\
\text { of } 24 \mathrm{mg} / \mathrm{d} \text {, no } \\
\text { granuloma seen at } \\
130 \text { weeks }\end{array}$ \\
\hline $\begin{array}{l}\text { Penn and } \\
\text { Paice } 1987 \\
\text { (81) }\end{array}$ & \begin{tabular}{|l|}
35 patients with \\
intractable cancer \\
pain, implanted \\
with IDDS, 8 with \\
nonmalignant pain
\end{tabular} & $\begin{array}{l}\text { Intrathecal } \\
\text { morphine infusion }\end{array}$ & $\begin{array}{l}\text { Relief measured } \\
\text { by self-reported } \\
\text { NRS, enteral opioid } \\
\text { consumption, } \\
\text { self-report increase } \\
\text { daily activities, } \\
\text { mean follow-up } 5.4 \\
\text { months for cancer } \\
\text { patients }\end{array}$ & $\begin{array}{l}17 / 35 \text { patients reported } \\
0-3 \text { on NRS and }>50 \% \\
\text { reduction of opioids with } \\
\text { significant increase activities, } \\
11 \text { reported VAS } 4-6 \text { with }< \\
50 \% \text { decrease opioids and } \\
\text { some activity improvement; } \\
80 \% \text { of patients had either } \\
\text { good or excellent relief }\end{array}$ & $\begin{array}{l}\text { Data supports } \\
\text { use of chronic } \\
\text { IT morphine } \\
\text { for treatment } \\
\text { of intractable } \\
\text { malignant pain }\end{array}$ & $\begin{array}{l}\text { No associated } \\
\text { morbidity or } \\
\text { mortality was } \\
\text { associated with } \\
\text { pump implantation } \\
\text { or infusion. }\end{array}$ \\
\hline
\end{tabular}

IDDS = Intrathecal drug delivery system; CMM = Conservative Medical Management; VRS = Verbal Rating Scale; NAS = Numeric Analog Scale; $\underline{\text { NRS }=\text { Numeric Rating Scale; IT }=\text { Intrathecal }}$ 
Intrathecal Therapies for Cancer and Non-Cancer Pain

Table 9. Summary description of observational studies for intrathecal drug delivery in non-cancer pain.

\begin{tabular}{|c|c|c|c|c|c|c|}
\hline $\begin{array}{l}\text { Study / } \\
\text { Methods }\end{array}$ & Participants & Intervention & Outcome & Results & $\begin{array}{c}\text { Conclusion }(\mathrm{s}) \\
\text { Short-term }<1 \\
\text { year } \\
\text { Long-term } \geq 1 \\
\text { year }\end{array}$ & Complications \\
\hline $\begin{array}{l}\text { Atli et al, } \\
2010(68)\end{array}$ & $\begin{array}{l}57 \text { patients spanning } \\
5 \text { diagnostic } \\
\text { groups: FBSS (28), } \\
\text { neuropathic pain } \\
\text { (16), malignancy } \\
\text { (2), visceral pain (5), } \\
\text { miscellaneous (6), } 49 \\
\text { included in study, } 43 \\
\text { at } 3 \text { year follow-up }\end{array}$ & $\begin{array}{l}\text { Implantation of } \\
\text { IDDS for pain }\end{array}$ & $\begin{array}{l}\text { Self-reported } \\
\text { pain scales (VAS), } \\
\text { complications, IT, } \\
\text { and systemic opioid } \\
\text { consumption }\end{array}$ & $\begin{array}{l}\text { VAS reduced } 7.7 \text { to } 5.7 \text { at } 3 \\
\text { years, along with systemic } \\
\text { opioid consumption mean } \\
\text { from } 183 \mathrm{mg} / \text { day morphine } \\
\text { equivalents to } 57.6 \mathrm{mg} / \\
\text { day at } 3 \text { years, along with } \\
\text { gradual increases in IT doses } \\
\text { of } 6.5 \mathrm{mg} / \text { day to } 12.2 \mathrm{mg} / \\
\text { day, pre-implant opioid } \\
\text { consumption inversely } \\
\text { correlated with treatment } \\
\text { success }\end{array}$ & $\begin{array}{l}\text { ITP therapy } \\
\text { produces long- } \\
\text { term reductions } \\
\text { in pain severity } \\
\text { scores and } \\
\text { oral opioids } \\
\text { consumption. }\end{array}$ & $\begin{array}{l}\text { Complication } \\
\text { rate } 20 \%, 14 \\
\text { complications } \\
\text { occurred in } 10 \\
\text { of } 57 \text { patients: } \\
5 \text { with wound } \\
\text { infection, } 2 \text { with IT } \\
\text { granuloma, } 2 \text { with } \\
\text { seroma at pump } \\
\text { site, } 3 \text { with catheter } \\
\text { migration fracture. } \\
\text { Treatment failure } \\
\text { rate } 24 \% \text {. }\end{array}$ \\
\hline $\begin{array}{l}\text { Duse et al } \\
2009(20)\end{array}$ & $\begin{array}{l}30 \text { patients with } \\
\text { chronic nociceptive, } \\
\text { neuropathic or mixed } \\
\text { non-cancer pain that } \\
\text { failed multimodal } \\
\text { analgesic regimens } \\
\text { (opioids, neuropathic } \\
\text { pain medications, } \\
\text { NSAIDs), patients } \\
\text { had pain for at least } \\
30 \text { months, average } \\
\text { age } 64 \text { year old, } \\
\text { weaned off opioids } \\
\text { prior to trialing with } \\
\text { epidural morphine } \\
\text { x } 3 \text { wks }\end{array}$ & $\begin{array}{l}\text { IDDS therapy with } \\
\text { morphine with } \\
\text { dose adjustment } \\
\text { to maintain }>50 \% \\
\text { reduction of initial } \\
\text { value }\end{array}$ & $\begin{array}{l}\text { At } 0,3,12 \text {, and } 24 \\
\text { month intervals } \\
\text { after implant and } \\
\text { evaluated by self- } \\
\text { reported VAS, MPQ }\end{array}$ & $\begin{array}{l}\text { MPQ improved } 66 \% \text {, } \\
\text { theeffective component } \\
59 \% \text {, and the sensory } \\
\text { component } 32 \% \text {. VAS } \\
\text { improved by } 55 \% \text {. The } \\
\text { average morphine infusion } \\
\text { rate increased to } 0.80 \pm 0.45 \\
\text { mg/day at the } 24 \text {-mo } \\
\text { follow-up intervals (P _ } \\
0.05 \text { ). Among } 13 \text { patients of } \\
\text { working age, } 12 \text { returned to } \\
\text { work full time. }\end{array}$ & $\begin{array}{l}\text { IT infusion of } \\
\text { morphine using } \\
\text { IDDS was helpful } \\
\text { in improving } \\
\text { psychosocial } \\
\text { function and } \\
\text { improved pain } \\
\text { scores in patients } \\
\text { that have failed } \\
\text { multimodal } \\
\text { therapy. }\end{array}$ & None reported \\
\hline $\begin{array}{l}\text { Ellis et al, } \\
2008(72)\end{array}$ & $\begin{array}{l}155 \text { patients with } \\
\text { severe chronic pain } \\
\text { (107 non-cancer } \\
\text { pain and } 48 \text { with } \\
\text { cancer related pain) } \\
\text { from one of } 2 \text { RCT } \\
\text { demonstrating } \\
\text { response; } 31 \text { patients } \\
\text { participated in study } \\
>12 \text { months, mean } \\
\text { was } 288 \text { days }\end{array}$ & $\begin{array}{l}\text { Implanted infusion } \\
\text { systems and external } \\
\text { micro infusion } \\
\text { pumps for cancer } \\
\text { pain patients. } \\
\text { Ziconotide only } \\
\text { therapy offered. }\end{array}$ & $\begin{array}{l}\text { At } 1,6,12 \text { months } \\
\text { functional capacity } \\
\text { and QOL scores } \\
\text { (PDI and SIP-20 } \\
\text { for patients from } \\
\text { non-cancer pain) } \\
\text { RTC vs. Karnofsky } \\
\text { Performance at } \\
\text { screening, twice } \\
\text { during first } 30 \\
\text { days, and then } \\
\text { monthly for cancer } \\
\text { pain; PDI, SIP-20 } \\
\text { and VASPI for } \\
\text { descriptive statistics; } \\
\text { safety analysis } \\
\text { was descriptive } \\
\text { COSTART for AE }\end{array}$ & $\begin{array}{l}61 / 155(39.4 \%) \text { stopped } \\
\text { because of AE, } 31 / 155 \text { were } \\
\text { followed for at least a year, } \\
\text { post-hoc analysis of retained } \\
\text { patients demonstrated no } \\
\text { attenuation of analgesic affect } \\
\text { and stable dose through } 12 \\
\text { months }(\mathrm{P}<0.0001) \text {; PDI and } \\
\text { SIP-20 not carried beyond } \\
\text { one-month; VASPI went } \\
\text { from } 77.9 \text { to } 43.6 \text { with mean } \\
\% \text { reduction of } 45.8\end{array}$ & $\begin{array}{l}\text { Post-hoc analysis } \\
\text { of retained patients } \\
\text { demonstrated } \\
\text { no attenuation of } \\
\text { analgesic effect of } \\
\text { mean \% reduction } \\
\text { of VASPI by } \\
45.8 \text { and stable } \\
\text { dose through } \\
12 \text { months } \\
(\mathrm{P}<0.0001) \text {; }\end{array}$ & $\begin{array}{l}\text { Ziconotide related } \\
\text { AE occurred } \\
\text { in } 147 / 155 \\
\text { patients; } 39.4 \% \\
\text { discontinued } \\
\text { treatment because } \\
\text { of AE, most } \\
\text { common being } \\
\text { confusion at } 43.2 \%\end{array}$ \\
\hline $\begin{array}{l}\text { Ilias et al } \\
2008(21)\end{array}$ & $\begin{array}{l}168 \text { patients with } \\
\text { existing ( } 79 \text { patients) } \\
\text { or recently implanted } \\
\text { IDDS ( } 89 \text { patients) } \\
\text { with unpredictable } \\
\text { pain fluctuations with } \\
\text { non-cancer ( } 92 \% \text {, } \\
\text { most common FBSS) } \\
\text { and cancer pain }(8 \%) \text {, } \\
\text { mean age } 56.4 \text { years, } \\
58 \% \text { female, } 119 \\
\text { patients had data at } 12 \\
\text { months }\end{array}$ & $\begin{array}{l}\text { Given personal } \\
\text { therapy manager } \\
\text { (PTM) options via } \\
\text { SynchroMed EL } \\
\text { or SynchroMed II } \\
\text { pumps }\end{array}$ & $\begin{array}{l}\text { Baseline visit, } \\
\text { screening and } \\
\text { implant visit for } \\
\text { patients without } \\
\text { previous IDDS, } \\
\text { a PTM start visit, } \\
\text { and follow-up at } 12 \\
\text { months measuring } \\
\text { VAS, EQ-5D, } \\
\text { medications, VAS, } \\
\text { PTM settings, } \\
\text { adverse events, } \\
\text { and self report } \\
\text { satisfaction }\end{array}$ & $\begin{array}{l}\text { At } 12 \text { months, patients with } \\
\text { existing IDDS, VAS decreased } \\
\text { from } 6.4 \text { to } 5.5 \text { at } 12 \text { months, } \\
\text { in newly implanted group, } \\
\text { VAS decreased from } 8.0 \\
\text { to } 4.1 \text {. Most common IT } \\
\text { drug was morphine ( } 66 \% \\
\text { at } 12 \text { months), PTM at } 12 \\
\text { months was } 170 \text { sec, lockout } \\
2 \text { hours, max/day } 5 \text { boluses, } \\
\text { average PTM per days } 1.3 \\
\text { at } 12 \text { mo; all patients tended } \\
\text { to decrease the concomitant } \\
\text { pain medication and the } \\
\text { QOL tended to improve (10\% } \\
\text { on the EQ-5D scale). In total, } \\
85 \% \text { of patients were satisfied } \\
\text { with the PTM device. }\end{array}$ & $\begin{array}{l}\text { Patient controlled } \\
\text { analgesia with } \\
\text { PTM is viable and } \\
\text { effective method } \\
\text { to treat patients } \\
\text { with intractable } \\
\text { pain and improves } \\
\text { control over } \\
\text { unpredictable pain } \\
\text { fluctuations. }\end{array}$ & $\begin{array}{l}181 \text { complications } \\
\text { occurred in } 92 \\
\text { patients, } 32 \% \\
\text { were benign and } \\
\text { drug related side- } \\
\text { effects, } 16 \text { were } \\
\text { related to pump } \\
\text { related events } \\
\text { that resolved after } \\
\text { reprogramming. } \\
\text { No serious adverse } \\
\text { events occurred. }\end{array}$ \\
\hline
\end{tabular}


Table 9 cont. Summary description of observational studies for intrathecal drug delivery in non-cancer pain.

\begin{tabular}{|c|c|c|c|c|c|c|}
\hline $\begin{array}{c}\text { Study I } \\
\text { Methods }\end{array}$ & Participants & Intervention & Outcome & Results & $\begin{array}{c}\text { Conclusion(s) } \\
\text { Short-term }<1 \\
\text { year } \\
\text { Long-term } \geq 1 \\
\text { year }\end{array}$ & Complications \\
\hline $\begin{array}{l}\text { Shaladi et al, } \\
2007 \text { (76) }\end{array}$ & $\begin{array}{l}24 \text { patients with } \\
\text { osteoporosis with } \\
\text { presence of chronic } \\
\text { vertebral compression } \\
\text { fracture, VAS }>7 \text { after } \\
\text { failed conservative } \\
\text { therapy for } 3 \text { months, } \\
\text { failed systemic } \\
\text { opioid therapy, drug } \\
\text { addition, and absence } \\
\text { of barriers with } \\
\text { successful trial }\end{array}$ & $\begin{array}{l}\text { IT morphine } \\
\text { after successful } \\
\text { trial of }>50 \% \text { pain } \\
\text { reduction with trial } \\
\text { of morphine ( } 0.5 \text { to } \\
1 \mathrm{mg} \text { ) }\end{array}$ & $\begin{array}{l}\text { VAS, QUALEFFO } \\
\text { (Quality of Life } \\
\text { Questionnaire } \\
\text { of the European } \\
\text { Foundation of } \\
\text { Osteoporosis), and } \\
\text { morphine dose } \\
\text { at one year post } \\
\text { implant }\end{array}$ & $\begin{array}{l}\text { VAS declined from } 8.7 \\
\text { pretrial to } 1.9 \text { one year later, } \\
\text { the QUALEFFO dropped } \\
\text { from } 114.7 \text { to } 79.1 \text { one year } \\
\text { post implant. Mean IT } \\
\text { morphine dose at one year } \\
\text { was } 16.32 \mathrm{mg} / \text { day. Patients } \\
\text { reported improved function } \\
\text { and satisfaction with therapy, } \\
\text { also no systemic opioid } \\
\text { medications }\end{array}$ & $\begin{array}{l}\text { IT morphine } \\
\text { relieves pain } \\
\text { and improves } \\
\text { QOL, in patients } \\
\text { with severe } \\
\text { osteoporosis. }\end{array}$ & $\begin{array}{l}\text { One wound } \\
\text { infection, one } \\
\text { delayed wound } \\
\text { healing, nausea, } \\
\text { and itching }\end{array}$ \\
\hline $\begin{array}{l}\text { Staats et al, } \\
2007 \text { (77) }\end{array}$ & $\begin{array}{l}101 \text { consecutive } \\
\text { patients with IDDS } \\
\text { from } 8 \text { different } \\
\text { centers (no more } \\
\text { than } 20 \text { per center) } \\
\text { implanted for } \\
\text { management of } \\
\text { chronic intractable } \\
\text { non-cancer back pain } \\
\text { with therapy for at } \\
\text { least } 2 \text { years }\end{array}$ & $\begin{array}{l}\text { IDDS therapy } \\
\text { with opioids, } \\
\text { local anesthetics, } \\
\text { adjuvants, or } \\
\text { combination thereof } \\
\text { for at least } 2 \text { years }\end{array}$ & $\begin{array}{l}\text { Outcomes were } \\
\text { time to initiation } \\
\text { of constant flow } \\
\text { treatment (either } \\
\text { simple continuous, } \\
\text { single bolus + } \\
\text { simple continuous), } \\
\text { number of program } \\
\text { adjustments, } \\
\text { medications used }\end{array}$ & $\begin{array}{l}\text { Most pumps had morphine } \\
(47.7 \%) \text {; pre-implant pain } \\
\text { score in } 89 \text { patients was } 7.7 \text {, } \\
\text { refill visits averaged every } \\
1.5 \text { months, } 34 \% \text { had single } \\
\text { medication therapy, } 35.6 \% \\
\text { had } 2 \text { medication therapy, } \\
89 \% \text { had daily morphine no } \\
\text { greater than } 25 \mathrm{mg} / \text { day, } 56.9 \% \\
\text { had concentration of }<25 \mathrm{mg} / \\
\text { cc; } 94 \% \text { had constant flow } \\
\text { treatment, maintained from } \\
1-3 \text { months to }>30 \text { months } \\
\text { post implant (mean initiating } \\
\text { was } 2.7 \text { month) }\end{array}$ & $\begin{array}{l}\text { Data suggests that } \\
\text { patients with non- } \\
\text { cancer pain could } \\
\text { be implanted with } \\
\text { constant flow rate } \\
\text { pump and can } \\
\text { be maintained } \\
\text { throughout } \\
\text { treatment }\end{array}$ & None reported \\
\hline $\begin{array}{l}\text { Doleys et al, } \\
2006(70)\end{array}$ & $\begin{array}{l}\text { Patients with non- } \\
\text { cancer pain, primarily } \\
\text { in lumbar back and } \\
\text { /or legs s/p FBSS, } \\
\text { with pretreatment } \\
\text { pain at least } 2 \text { years in } \\
\text { duration, intrathecal } \\
\text { therapy ( } 50 \text { ) after } \\
2 \text { week epidural } \\
\text { trial with at least } \\
50 \% \text { improvement } \\
\text { in pain, systemic } \\
\text { opioid therapy ( } 40 \text { ), } \\
\text { and residential } \\
\text { pain/rehabilitation } \\
\text { program (40), all of } \\
\text { which resided within } \\
\text { Birmingham, AL. }\end{array}$ & $\begin{array}{l}\text { IT therapy was } \\
\text { morphine, } \\
\text { hydromorphone, } \\
\text { or fentanyl } \pm \text { local } \\
\text { anesthetic }\end{array}$ & $\begin{array}{l}\text { NRS, ODQ, } \\
\text { Beck Depression } \\
\text { Inventory, MPQ, } \\
\text { health related QOL, } \\
\text { and SF-36, work } \\
\text { status, and patient } \\
\text { opinion measures } \\
\text { for } 4.2 \text { years (IDDS), } \\
4.3 \text { years (rehab), } \\
\text { and } 4.8 \text { years } \\
\text { (systemic opioid } \\
\text { therapy) }\end{array}$ & $\begin{array}{l}\text { The IDDS group appeared } \\
\text { superior to the other } 2 \\
\text { groups, with the NRS with } \\
\text { the greatest change ( } 7.9 \text { to } \\
5.12 \text { ), while the systemic } \\
\text { opioid group reported overall } \\
\text { satisfaction better while the } \\
\text { rehab group reported highest } \\
\text { quality of well being score. }\end{array}$ & $\begin{array}{l}\text { There appeared } \\
\text { to be a disconnect } \\
\text { between QOL, } \\
\text { pain, disability, } \\
\text { and mood, and } \\
\text { the IDDS group } \\
\text { appeared o } \\
\text { report greatest } \\
\text { improvement. }\end{array}$ & None reported \\
\hline $\begin{array}{l}\text { Thimineur } \\
\text { et al, } \\
2004 \text { (78) }\end{array}$ & $\begin{array}{l}69 \text { non-cancer } \\
\text { pain patients with } \\
\text { pain determined as } \\
\text { severe, conservative } \\
\text { treatment failure, } \\
\text { divided into } 2 \text { groups. } \\
38 \text { pts. received } \\
\text { intrathecal pump, } 31 \\
\text { did not (patients with } \\
\text { unsuccessful trial or } \\
\text { declined intrathecal } \\
\text { therapy) and were } \\
\text { followed as NR. } \\
\text { Another group of new } \\
\text { patients (n }=41 \text { ) used } \\
\text { as comparative group } \\
\text { NR (non-recipient } \\
\text { group). }\end{array}$ & $\begin{array}{l}\text { Intrathecal } \\
\text { morphine, } \\
\text { hydromorphone, } \\
\text { fentanyl, Clonidine, } \\
\text { Baclofen, } \\
\text { bupivacaine, and } \\
\text { methadone. Non } \\
\text { intrathecal group } \\
\text { continued the pre- } \\
\text { study medications } \\
\text { (systemic opioids). }\end{array}$ & $\begin{array}{l}\text { Multiple } \\
\text { questionnaires } \\
\text { - symptom } \\
\text { checklist 90-R, } \\
\text { SF-36 Health study, } \\
\text { Beck depression } \\
\text { questionnaire, MPQ } \\
\text { - short, Oswestry } \\
\text { disability index, } \\
\text { Pain drawing, VAS } \\
\text { (1-10). Evaluations } \\
\text { done at baseline and } \\
\text { then every } 6 \text { months } \\
\text { for } 3 \text { years. }\end{array}$ & $\begin{array}{l}\text { Intrathecal treatment had } \\
\text { a significant impact on } \\
\text { pain, function, and mood } \\
\text { among study patients. Non- } \\
\text { recipients deteriorated despite } \\
\text { escalation of oral opioids } \\
\text { and provision of injection } \\
\text { treatments. The baseline } \\
\text { opioid requirements were } \\
\text { higher in the pump recipients } \\
\text { (PR) than non-recipients } \\
\text { (NR). At } 36 \text { months, the } \\
\text { average daily oral morphine } \\
\text { dose had significantly } \\
\text { decreased for PR group and } \\
\text { increased for NR. }\end{array}$ & $\begin{array}{l}\text { Intrathecal opioid } \\
\text { therapy for non- } \\
\text { cancer pain should } \\
\text { be considered } \\
\text { appropriate only } \\
\text { when all other } \\
\text { conservative } \\
\text { medical } \\
\text { management } \\
\text { options have been } \\
\text { exhausted. }\end{array}$ & $\begin{array}{l}\text { Pocket infection in } \\
\text { 2, one had kinking } \\
\text { of catheter; one } \\
\text { patient had } \\
\text { transverse myelitis } \\
\text { picture with } \\
\text { continued motor } \\
\text { deficiency }\end{array}$ \\
\hline
\end{tabular}


Table 9 cont. Summary description of observational studies for intrathecal drug delivery in non-cancer pain.

\begin{tabular}{|c|c|c|c|c|c|c|}
\hline $\begin{array}{c}\text { Study I } \\
\text { Methods }\end{array}$ & Participants & Intervention & Outcome & Results & $\begin{array}{c}\text { Conclusion(s) } \\
\text { Short-term }<1 \\
\text { year } \\
\text { Long-term } \geq 1 \\
\text { year }\end{array}$ & Complications \\
\hline $\begin{array}{l}\text { Deer et al, } \\
2004 \text { (22) }\end{array}$ & $\begin{array}{l}\text { Multicenter } \\
\text { prospective registry } \\
\text { of } 136 \text { patients with } \\
\text { chronic low back pain } \\
>\text { leg pain }\end{array}$ & $\begin{array}{l}\text { Implantable drug } \\
\text { infusion systems } \\
\text { delivering opioid } \\
\text { following successful } \\
\text { trial. } 81.1 \% \text { were } \\
\text { trialed with } \\
\text { morphine only }\end{array}$ & $\begin{array}{l}\text { Outcome measures: } \\
\text { Numeric Pain } \\
\text { Ratings and } \\
\text { Oswestry scores, } \\
\text { secondary } \\
\text { outcomes amount } \\
\text { of medications via } \\
\text { other routes and } \\
\text { return to work }\end{array}$ & $\begin{array}{l}\text { Significant improvements } \\
\text { in numeric pain ratings and } \\
\text { Oswestry scores at } 6 \text { and } 12 \\
\text { months. } \\
\text { Few patients who had } \\
\text { successful trials but were not } \\
\text { implanted did not improve. } \\
\text { Patients with neuropathic } \\
\text { pain had a statistically } \\
\text { significantly lower trial } \\
\text { success rate. } \\
\text { There was a decrease in oral } \\
\text { opioids and maintenance or } \\
\text { improvement in work status } \\
\text { in implanted patients. }\end{array}$ & $\begin{array}{l}\text { At } 12 \text { months, } \\
\text { patients implanted } \\
\text { had significant } \\
\text { improvement in } \\
\text { pain and Oswestry } \\
\text { scores and a high } \\
\text { satisfaction rate }\end{array}$ & $\begin{array}{l}23 \text { patients had } \\
\text { adverse events } \\
\text { with IIDS, } 21 \\
\text { required surgery } \\
\text { for correction: } \\
\text { infection }(2.2 \%) \text {, } \\
\text { migration }(1.5 \%) \text {, } \\
\text { CSF leak }(0.7 \%) \text {, } \\
\text { catheter kinking } \\
(1.5 \%) \text {, and } \\
\text { catheter fracture } \\
\text { (0.7\%), reaction } \\
\text { to medication } \\
\text { (5.1\%).All resolved } \\
\text { without untoward } \\
\text { events. }\end{array}$ \\
\hline $\begin{array}{l}\text { Deer et al, } \\
2002(69)\end{array}$ & $\begin{array}{l}109 \text { consecutive } \\
\text { patients for } \\
\text { bupivacaine }+ \text { opioid } \\
\text { compared with opioid } \\
\text { alone, } 84 \text { non-cancer } \\
\text { patients and } 25 \text { cancer } \\
\text { patients }\end{array}$ & $\begin{array}{l}\text { Implantable drug } \\
\text { infusion systems } \\
\text { delivering opioid } \\
\text { alone vs opioid }+ \\
\text { bupivacaine }\end{array}$ & $\begin{array}{l}\text { Primary outcome } \\
\text { measure - pain } \\
\text { relief via VAS } \\
\text { score, secondary } \\
\text { outcomes amount } \\
\text { of medications via } \\
\text { other routes (oral/ } \\
\text { transdermal), } \\
\text { ER visits, routine } \\
\text { office visits, patient } \\
\text { satisfaction. } \\
\text { Neurological } \\
\text { complications } \\
\text { reviewed with } \\
\text { combined drugs. }\end{array}$ & $\begin{array}{l}\text { With combination } \\
\text { (bupivacaine + opioid) } \\
\text { infusion the pain relief } \\
\text { was significantly better, } \\
\text { the number of oral opioids } \\
\text { used were significantly less, } \\
\text { number of oral non-opioid } \\
\text { adjuvants were reduced, } \\
\text { number of doctor's visits } \\
\text { were less in the combined } \\
\text { arm, number of pain clinic } \\
\text { visits were less, the number } \\
\text { of emergency visits were } \\
\text { significantly less, and patient } \\
\text { satisfaction was better. } \\
\text { Total dose of morphine } \\
\text { was reduced by } 23 \% \text { with } \\
\text { combined drugs. }\end{array}$ & $\begin{array}{l}\text { Bupivacaine, } \\
\text { when used in } \\
\text { combination with } \\
\text { opioids, is a helpful } \\
\text { and safe method } \\
\text { of treatment in a } \\
\text { select population } \\
\text { of patients } \\
\text { who have not } \\
\text { responded to } \\
\text { intrathecal opioids } \\
\text { alone. }\end{array}$ & $\begin{array}{l}\text { Medication } \\
\text { related side } \\
\text { effects, including } \\
\text { non-dermatomal } \\
\text { numbness, } \\
\text { peripheral edema, } \\
\text { summarily: no } \\
\text { long-term effects } \\
\text { associated with IT } \\
\text { therapy }\end{array}$ \\
\hline $\begin{array}{l}\text { Dominguez } \\
\text { et al, } 2002 \\
\text { (71) }\end{array}$ & $\begin{array}{l}\text { Retrospective study of } \\
\text { implanted patients at } \\
\text { a tertiary center. } 157 \\
\text { patients screened, } 134 \\
\text { implanted, of whom } \\
86 \text { were available for } \\
\text { follow up }\end{array}$ & $\begin{array}{l}\text { All patients received } \\
\text { IT bolus trials with } \\
\text { morphine starting at } \\
0.5 \mathrm{mg} \text {. Those who } \\
\text { did not respond, } \\
\text { were given } 1 \mathrm{mg} \\
\text { or more boluses } \\
\text { (high responders). } \\
\text { Patients were } \\
\text { implanted with } \\
\text { morphine initially. }\end{array}$ & $\begin{array}{l}\text { Dose escalation } \\
\text { comparison between } \\
\text { high responders } \\
\text { vs. standard/low } \\
\text { dose responders, } \\
\text { dose escalation with } \\
\text { respect to gender, } \\
\text { age, and underlying } \\
\text { pain condition }\end{array}$ & $\begin{array}{l}\text { High responders had a } \\
\text { disproportionate increase } \\
\text { in opioid requirements. } \\
\text { Hydromorphone replaced } \\
\text { morphine or adjuvants were } \\
\text { added in half the cases by } \\
18 \text { months. Women and } \\
\text { individuals older than } 65 \\
\text { years had lower opioid } \\
\text { requirements } 18 \text { months or } \\
\text { more post-implant. }\end{array}$ & $\begin{array}{l}\text { The degree of } \\
\text { responsiveness } \\
\text { to an intrathecal } \\
\text { narcotic during a } \\
\text { trial period, along } \\
\text { with the patient's } \\
\text { age and gender, } \\
\text { have predictive } \\
\text { value on the long- } \\
\text { term utilization } \\
\text { of IT analgesics } \\
\text { in chronic non- } \\
\text { cancer patients. }\end{array}$ & $\begin{array}{l}\text { Poor patient } \\
\text { tolerance of the } \\
\text { implantable device, } \\
\text { intolerable side } \\
\text { effects, infection, } \\
\text { or failure to } \\
\text { achieve acceptable } \\
\text { pain relief led } \\
\text { to removal of } \\
\text { the device in } \\
17 \text { patients of } \\
\text { the original } 134 \\
\text { implanted. } 31 \\
\text { patients followed } \\
\text { up care elsewhere. }\end{array}$ \\
\hline $\begin{array}{l}\text { Rainov et al, } \\
2001 \text { (73) }\end{array}$ & $\begin{array}{l}26 \text { patients, median } \\
\text { age } 54 \text { years, s/p } \\
\text { FBSS and failed } \\
\text { conservative } \\
\text { management; } \\
18 \text { of which had } \\
\text { neuropathic/ } \\
\text { nociceptive pain, } \\
6 \text { with radicular, } \\
\text { and } 2 \text { with } \\
\text { radicular peripheral } \\
\text { neuropathic; } \\
\text { nondermatomal }\end{array}$ & $\begin{array}{l}\text { Intrathecal } \\
\text { morphine/ clonidine } \\
\text { or morphine/ } \\
\text { bupivacaine or } \\
\text { morphine/ } \\
\text { bupivacaine/ } \\
\text { clonidine, or } \\
\text { morphine/ } \\
\text { clonidine/ } \\
\text { bupivacaine/ } \\
\text { midazolam }\end{array}$ & $\begin{array}{l}\text { Mean follow-up was } \\
\text { up to } 27 \text { months, } \\
\text { data collected } 2 \\
\text { years: VAS, and non- } \\
\text { formal self-report } \\
\text { of daily activities, } \\
\text { including walking, } \\
\text { sleep, systemic } \\
\text { medications, motor } \\
\text { disturbances, } \\
\text { sensory } \\
\text { disturbances, and } \\
\text { bladder control, } \\
\text { dose of IT morphine } \\
\text { per day, and patient } \\
\text { global rating of } \\
\text { therapy }\end{array}$ & $\begin{array}{l}\text { VAS maintained at one } \\
\text { month following implant } \\
\text { to } 2 \text { years from } 8 \text { to } 50 \% \\
\text { improvement; most patients } \\
\text { reported improvement in } \\
\text { walking ability, reduction } \\
\text { systemic pain medications, } \\
\text { and sleep; mean daily } \\
\text { dose of morphine alone } \\
\text { or in combination was } 6.2 \\
\pm 2.8 \mathrm{mg} \text { morphine, } 2.5 \mathrm{mg} \\
\pm 1.5 \mathrm{mg} \text { bupivacaine, } 0.06 \mathrm{mg} \\
\pm 0.03 \mathrm{mg} \text { for clonidine, } \\
\text { and } 0.8 \mathrm{mg} \pm 0.4 \mathrm{mg} \text { for } \\
\text { midazolam; } 73 \% \text { rated long- } \\
\text { term treatment as excellent } \\
\text { or good. }\end{array}$ & $\begin{array}{l}\text { Intrathecal } \\
\text { polyanalgesia } \\
\text { employing } \\
\text { morphine alone } \\
\text { or in combination } \\
\text { with non-opioid } \\
\text { drugs can have } \\
\text { a favorable and } \\
\text { sustained } \\
\text { analgesic efficacy } \\
\text { in patients with } \\
\text { complex chronic } \\
\text { pain of spinal } \\
\text { origin, with lack } \\
\text { of major } \\
\text { drug-related } \\
\text { complications. }\end{array}$ & $\begin{array}{l}3 / 26 ; 2 \text { device } \\
\text { occlusion of } \\
\text { catheter and } \\
\text { leakage; one } \\
\text { human error } \\
\text { during refilling } \\
\text { causing leak of } \\
\text { medication from } \\
\text { reservoir septum }\end{array}$ \\
\hline
\end{tabular}


Table 9 cont. Summary description of observational studies for intrathecal drug delivery in non-cancer pain.

\begin{tabular}{|c|c|c|c|c|c|c|}
\hline $\begin{array}{c}\text { Study / } \\
\text { Methods }\end{array}$ & Participants & Intervention & Outcome & Results & $\begin{array}{c}\text { Conclusion(s) } \\
\text { Short-term <1 } \\
\text { year } \\
\text { Long-term } \geq \mathbf{1} \\
\text { year }\end{array}$ & Complications \\
\hline $\begin{array}{l}\text { Roberts } \\
\text { et al, } \\
2001 \text { (75) }\end{array}$ & $\begin{array}{l}88 \text { patients with } \\
\text { implanted drug adm. } \\
\text { systems (1989-1996). } \\
\text { Diagnoses included } \\
\text { failed spinal surgery } \\
(\mathrm{n}=55) \text {, lumbar } \\
\text { spinal or radicular } \\
\text { pain without surgery } \\
(\mathrm{n}=6), \text { CRPS I }(\mathrm{n}=5) \text {, } \\
\text { cervical failed spinal } \\
\text { surgery }(\mathrm{n}=4) \text {, crush } \\
\text { fractures }(\mathrm{n}=3) \text {, } \\
\text { chronic pancreatitis } \\
(\mathrm{n}=3) \text {, others }(\mathrm{n} \\
=12) .\end{array}$ & $\begin{array}{l}\text { Intrathecal opioids } \\
\text { (morphine) via } \\
\text { implantable drug } \\
\text { administrations } \\
\text { systems after a } \\
\text { successful trial }\end{array}$ & $\begin{array}{l}\text { Global pain relief, } \\
\text { physical activity } \\
\text { levels, medication } \\
\text { consumption, work } \\
\text { status, intrathecal } \\
\text { opioid side-effects, } \\
\text { proportion of } \\
\text { patients who ceased } \\
\text { therapy, and patient } \\
\text { satisfaction }\end{array}$ & $\begin{array}{l}\text { Mean pain relief - } 60 \% \text { with } \\
74 \% \text { of patients ( } 36 \text { of } 49 \text { ) } \\
\text { reporting increased activity } \\
\text { levels. Significant reduction } \\
\text { in oral medications. Frequent } \\
\text { side effects such as sexual } \\
\text { dysfunction, menstrual } \\
\text { disturbance were reported. } \\
88 \% \text { patients reported high } \\
\text { satisfaction levels. Change in } \\
\text { work status was not seen in } \\
\text { the patients. }\end{array}$ & $\begin{array}{l}\text { Intrathecal opioid } \\
\text { therapy appears } \\
\text { to have a place in } \\
\text { the management } \\
\text { of chronic } \\
\text { non-cancer pain. } \\
\text { Therapy does } \\
\text { not seem to be } \\
\text { significantly } \\
\text { inhibited by the } \\
\text { development of } \\
\text { tolerance. }\end{array}$ & $\begin{array}{l}\text { Technical } \\
\text { complications } \\
\text { occurred } \\
\text { with the drug } \\
\text { administration } \\
\text { device, } \\
\text { predominantly } \\
\text { catheter related, } \\
\text { requiring at } \\
\text { least one further } \\
\text { surgical procedure } \\
\text { in } 32 \text { patients } \\
(40 \%) \text {. }\end{array}$ \\
\hline $\begin{array}{l}\text { Anderson } \\
\text { and } \\
\text { Burchiel, } \\
1999(67)\end{array}$ & $\begin{array}{l}40 \text { consecutive } \\
\text { patients with } \\
\text { non-cancer pain, } \\
58 \% \text { women, } 47 \% \\
\text { had FBSS; } 30 \text { had a } \\
\text { successful trial and } \\
\text { were implanted. } \\
50 \% \text { of patients had } \\
\text { mixed neuropathic- } \\
\text { nociceptive and } \\
33 \% \text { had peripheral } \\
\text { neuropathic pain }\end{array}$ & $\begin{array}{l}14 \text { patients were } \\
\text { screened by sihkijhkeln } \\
\text { injection of } 1996 \text { ( } 79 \\
\text { mg morphine } \\
\text { intrathecally. } 26 \\
\text { patients had } 2-3 \\
\text { day trial of epidural } \\
\text { morphine infusion } \\
\text { by external pump. } \\
\text { Patients with } \geq \\
50 \% \text { pain relief } \\
\text { were implanted } \\
\text { with IDDS } \\
\text { (10/14 and 20/26 } \\
\text { respectively) with } \\
\text { either morphine, } \\
\text { hydrocodone, or in } \\
\text { combination with } \\
\text { bupivacaine }\end{array}$ & 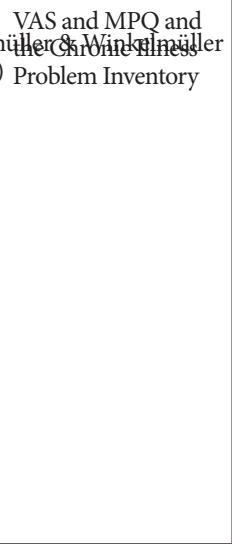 & $\begin{array}{l}\text { Data was available on } 20 \text { out } \\
\text { of the } 27 \text { surviving patients at } \\
24 \text { months. VAS scores were } \\
\text { significantly decreased over } \\
\text { baseline at each reporting } \\
\text { interval for the entire } 24 \\
\text { months of follow-up. } 50 \% \\
\text { of patients reported } \geq 25 \% \\
\text { pain relief at } 24 \text { months } \\
\text { post-implant. }\end{array}$ & $\begin{array}{l}\text { Continuous } \\
\text { intrathecal } \\
\text { morphine can be } \\
\text { a safe, effective, } \\
\text { and sustainable } \\
\text { therapy for the } \\
\text { management of } \\
\text { severe, non-cancer } \\
\text { pain among } \\
\text { carefully selected } \\
\text { patients. }\end{array}$ & $\begin{array}{l}\text { No infections. } \\
2 \text { patients had } \\
\text { PDPH, } 5 \text { had } \\
\text { device related } \\
\text { complications } \\
\text { requiring repeat } \\
\text { operation, pump } \\
\text { malfunction x2 } \\
\text { patients, one } \\
\text { programming } \\
\text { error, } \\
\text { pharmacologic } \\
\text { complications } \\
\text { resolved within } 3 \\
\text { months of therapy }\end{array}$ \\
\hline $\begin{array}{l}\text { Winkelmüller } \\
\text { \& } \\
\text { Winkelmüller } \\
1996 \text { (79) }\end{array}$ & $\begin{array}{l}120 \text { patients with } \\
\text { non-cancer pain } \\
\text { followed from } 6 \text { mos. } \\
\text { to } 5.7 \text { yrs. Patients } \\
\text { had nociceptive- } \\
\text { neuropathic pain due } \\
\text { to multiple lumbar } \\
\text { spinal operations. }\end{array}$ & $\begin{array}{l}\text { Intrathecal } \\
\text { morphine (+ } \\
\text { buprenorphine, } \\
\text { Clonidine, fentanyl, } \\
\text { or NaCl in various } \\
\text { combinations) } \\
\text { via implantable } \\
\text { pump. Additional } \\
\text { medications } \\
\text { were included as } \\
\text { a combination } \\
\text { and consisted } \\
\text { of bupivacaine, } \\
\text { clonidine, fentanyl, } \\
\text { and buprenorphine. }\end{array}$ & $\begin{array}{l}\text { Outcome } \\
\text { measurement with } \\
\text { VAS and MPQ, level } \\
\text { of activity, mood, } \\
\text { QOL, complications } \\
\text { and side effects }\end{array}$ & $\begin{array}{l}\text { Deafferentation pain and } \\
\text { neuropathic pain showed } \\
\text { the best long-term results, } \\
\text { with } 68 \% \text { and } 62 \% \text { pain } \\
\text { reduction. Pain reduction } \\
\text { after } 6 \text { months was } 67.4 \% \\
\text { and, as of the last follow-up } \\
\text { examination, it was } 58.1 \% \text {. } \\
92 \% \text { patients were satisfied } \\
\text { with the therapy and } 81 \% \\
\text { reported an improvement in } \\
\text { their QOL. VAS measured } \\
\text { pre-implant=93.6, } 6 \text { mo. } \\
\text { later VAS = 30.5, last f/u } \\
\text { VAS=39.2. Best initial } \\
\text { reduction in pain }(77 \%) \text { in } \\
\text { nociceptive group which } \\
\text { decreased to } 48 \% \text { at last f/u; } \\
\text { improved level of activity; } \\
67 \% \text { pts. satisfied with } \\
\text { pain level, } 81 \% \text { improved } \\
\text { QOL. Morphine was the } \\
\text { most effective and tolerated } \\
\text { substance. Complications: } \\
14 \text { pumps replaced, } 25 \\
\text { pumps removed (28.5\% pts } \\
\text { considered failures). }\end{array}$ & $\begin{array}{l}\text { Long-term } \\
\text { administration } \\
\text { of spinal opioid } \\
\text { medications for } \\
\text { non-cancer pain } \\
\text { is encouraging in } \\
\text { carefully selected } \\
\text { patients. Good } \\
\text { results were } \\
\text { achieved in a } \\
\text { total of } 74.2 \% \text { of } \\
\text { the patients, and } \\
\text { a pain reduction } \\
\text { of approximately } \\
60 \% \text { was reported } \\
\text { even after long- } \\
\text { term opioid } \\
\text { application. }\end{array}$ & $\begin{array}{l}14 \text { pumps had to } \\
\text { be replaced for } \\
\text { technical reasons, } \\
\text { including irregular } \\
\text { flow rates, skin } \\
\text { perforations, or } \\
\text { refilling errors; } \\
\text { in all } 31 \text { of } 120 \\
\text { were considered } \\
\text { treatment failures. }\end{array}$ \\
\hline
\end{tabular}

MPQ = McGill Pain Questionnaire; QOL = Qualityquality of Lifelife; FBSS= Failed Back Surgery Syndrome; VAS = Visual Analog Scale; ITP = Intrathecal Pump; IDDS = Intrathecal Drug Delivery System: IT = Intrathecal; AE = Adverse Events; NSAID = Non-Steroidal Anti-Inflammatory Drug; QOL = Quality of Life; SIP = Sickness Impact Profile; PDI = Pain Disability Index; VASPI = Visual Analog Scale of Pain Intensity; COSTART = Coding Symbols for Thesarus of Adverse Reaction Terms; PTM = Personal Therapy Manager; EQ-5D = EuroQoL Questionnaire; QUALEFFO = Quality of I ife Questionnaire of the European Foundation for Osteoporosis 
(20) prospectively evaluated the effects of chronic intrathecal morphine delivery on emotional variables affecting pain perception and functioning in patients with severe chronic non-cancer pain involving the low back and/or lower extremities. Of 42 patients evaluated, 30 were implanted with IDDS after a successful epidural morphine infusion trial. All patients were taking opioids prior to enrolling in the study and received only morphine intrathecally, were followed for 24 months, and were administered serial MPQ. VAS scores were significantly improved at all time intervals studied compared to baseline. Significant progressive improvements were noted in the affective, evaluative, sensory, and mixed components of the MPQ. Good to excellent satisfaction was reported in 29 of the 30 implanted patients; activities of daily living were improved in 26 patients and 12 patients were able to return to full-time employment. No significant complications were noted. A more recent study by Atli et al (68) retrospectively examined charts of 57 patients, 55 with non-cancer pain. About half had a diagnosis of "FBSS" and 16 patients had neuropathic pain. Patients were trialed using a continuous intrathecal infusion with morphine and infrequently with hydromorphone or sufentanil and titrated over a 5-day period. Patients were implanted with the same solution if they achieved satisfactory pain relief at the end of the trial period. Patients were followed for 3 years from implant. Eight patients were excluded; 3 because of death (including the 2 cancer pain patients), 2 for relocating to a different geographical area, 2 for pump infection/ revision, and one patient was excluded for being an "outlier." There was a statistically significant decrease in VAS pain scores at one, 2, and 3 years post-implant. However, a clear trend of temporal decrease in percentage of patients with $>50 \%$ pain relief and those with $>30 \%$ pain relief emerged, such that by the end of 3 years post-implant $18 \%$ of patients experienced $>50 \%$ pain relief and $37 \%$ had $>30 \%$ pain relief. However, oral opioid consumption was decreased significantly throughout the 3-year follow-up and $24 \%$ of patients had ceased all oral opioid consumption. Of note, higher initial oral opioid consumption was correlated with lower likelihood of long-term pain relief with intrathecal opioids. No adjuvant intrathecal medications were allowed and the intrathecal opioid requirements in morphine equivalents per 24-hour increased from 6.5 $\mathrm{mg}$ at baseline to $12.2 \mathrm{mg}$ at 3-years, an $88 \%$ increase. Complications included infection in 5 patients, 3 catheter revisions, 2 intrathecal granulomas, and 2 pocket seromas. Summary results of non-cancer pain studies are displayed in Table 10

Only 5 cancer-related pain studies were identified and the study characteristics are described here. Smith et al (83) performed a randomized, controlled study comparing comprehensive medical management (CMM) to IDDS for intractable mixed neuropathic and nociceptive refractory cancer related pain. Refractory cancer pain was defined as patients reporting a VAS score greater than or equal to 5 despite $200 \mathrm{mg} /$ day oral morphine equivalents. Success was defined as improvement in VAS or reduction in toxicity as primary outcomes; 60/71 IDDS patients achieved success, as compared to $51 / 72 \mathrm{CMM}$ patients. Further, this randomized trial demonstrated greater survival at 6 months in patients receiving IDDS. Drug related toxicities were less in the IDDS group compared to the CMM group and were especially significant for reductions in fatigue and depressed level of consciousness. A total of 194 serious complications were reported, split evenly between the 2 groups. Of the 99 complications in the IDDS arm, 16 were related to the "implanted pump or related procedure," 10 requiring revision and one requiring explant (83). A prospective cancer pain study by Rauck et al (74) followed patients for over a year. In that study, 119 patients were implanted with an investigational patient-activated internalized intrathecal morphine delivery system. This study showed that patients with inadequately controlled cancer pain or having intolerable side effects achieved better analgesia when managed with this patient-activated intrathecal morphine bolus delivery device. However, significant complications were noted related to device function and/or procedure and the device did not receive FDA approval (74). Additionally, of the 119 cancer pain patients implanted, only 15 made it to 13 months. Hence, for cancer pain studies, a lesser duration of follow up than one year is more reasonable. Becker et al (80) retrospectively reviewed 43 consecutive patients with neuropathic, nociceptive, and mixed cancer-related pain who were treated with intrathecal opioid therapy. Data regarding pain response via numerical rating scale, intrathecal dose, and pain relief relative to type and location of pain were collected. Neuropathic pain and nociceptive pain was reduced in initial stages, as measured by the numerical rating scale, of $61.1 \%$ and $77.8 \%$, respectively. It was noted that in later stages of the therapy, pain relief was significantly less in neuropathic pain than in nociceptive pain. Reported complications of IDDS were discovered and treated in 5 patients (none related to malfunction of hardware or local infection, 3 had spi- 
Pain Physician: May/June 2011; 14:219-248

Table 10. Summary results of eligible non-cancer pain studies included in this systematic review.

\begin{tabular}{|c|c|c|c|c|c|c|c|c|c|}
\hline \multirow[t]{2}{*}{ Study } & \multirow{2}{*}{$\begin{array}{c}\text { Study } \\
\text { Characteristics }\end{array}$} & \multirow{2}{*}{$\begin{array}{l}\text { Methodological } \\
\text { Quality Scoring }\end{array}$} & \multirow{2}{*}{$\begin{array}{l}\text { Number of } \\
\text { Participants }\end{array}$} & \multicolumn{2}{|c|}{$\geq 30 \%$ Pain Relief } & \multicolumn{2}{|c|}{$\geq 50 \%$ Pain Relief } & \multicolumn{2}{|c|}{ Results } \\
\hline & & & & 6 mos. & $\geq 12$ mos. & 6 mos. & $\geq 12$ mos. & $\begin{array}{l}\text { Short-term } \\
<6 \mathrm{mos}\end{array}$ & $\begin{array}{c}\text { Long-term } \geq \\
12 \mathrm{mos}\end{array}$ \\
\hline Atli et al, 2010 (68) & $\begin{array}{l}\text { Observational } \\
\text { Retrospective }\end{array}$ & 64 & 43 & & $\mathrm{x}$ & & & & $\mathrm{x}$ \\
\hline Duse et al, 2009 (20) & $\begin{array}{c}\text { Observational } \\
\text { Prospective }\end{array}$ & 66 & 30 & & & & $\mathrm{x}$ & & $\mathrm{x}$ \\
\hline Ellis et al, 2008 (72) & $\begin{array}{l}\text { Observational } \\
\text { Prospective }\end{array}$ & & 155 & & $\mathrm{x}$ & & & & $\mathrm{x}$ \\
\hline Ilias et al, 2008 (21) & $\begin{array}{l}\text { Observational } \\
\text { Prospective }\end{array}$ & 64 & 168 & & $\mathrm{x}$ & & & & $\mathrm{x}$ \\
\hline $\begin{array}{l}\text { Shaladi et al, } 2007 \\
\text { (76) }\end{array}$ & $\begin{array}{l}\text { Observational } \\
\text { Prospective }\end{array}$ & 63 & 24 & & & & $\mathrm{x}$ & & $\mathrm{x}$ \\
\hline $\begin{array}{l}\text { Staats et al, } 2007 \\
\text { (77) }\end{array}$ & $\begin{array}{l}\text { Observational } \\
\text { Retrospective }\end{array}$ & & 101 & & $\mathrm{x}$ & & & & $\mathrm{x}$ \\
\hline $\begin{array}{l}\text { Doleys et al, } 2006 \\
\text { (70) }\end{array}$ & $\begin{array}{l}\text { Observational } \\
\text { Retrospective }\end{array}$ & & 180 & & $\mathrm{x}$ & & & & $\mathrm{x}$ \\
\hline $\begin{array}{l}\text { Thimineur et al, } \\
2004 \text { (78) }\end{array}$ & $\begin{array}{l}\text { Observational } \\
\text { Prospective }\end{array}$ & 60 & 69 & & $\mathrm{x}$ & & & & $\mathrm{x}$ \\
\hline Deer et al, 2004 (22) & $\begin{array}{l}\text { Observational } \\
\text { Prospective }\end{array}$ & 65 & 136 & $\mathrm{x}$ & $\mathrm{x}$ & & & $\mathrm{x}$ & $\mathrm{x}$ \\
\hline Deer et al, 2002 (69) & $\begin{array}{l}\text { Observational } \\
\text { Retrospective }\end{array}$ & 53 & 109 & & & & $\mathrm{x}$ & & $\mathrm{x}$ \\
\hline $\begin{array}{l}\text { Dominguez et al, } \\
2002(71)\end{array}$ & $\begin{array}{l}\text { Observational } \\
\text { Retrospective }\end{array}$ & 62 & 86 & & & & $\mathrm{x}$ & & $\mathrm{x}$ \\
\hline $\begin{array}{l}\text { Rainov et al, } 2001 \\
\text { (73) }\end{array}$ & $\begin{array}{l}\text { Observational } \\
\text { Prospective }\end{array}$ & 50 & 26 & & & & $\mathrm{x}$ & & $\mathrm{x}$ \\
\hline $\begin{array}{l}\text { Roberts et al, } 2001 \\
\text { (75) }\end{array}$ & $\begin{array}{l}\text { Observational } \\
\text { Retrospective }\end{array}$ & 50 & 88 & & & & $\mathrm{x}$ & & $\mathrm{x}$ \\
\hline $\begin{array}{l}\text { Anderson and } \\
\text { Burchiel, } 1999 \text { (67) }\end{array}$ & $\begin{array}{l}\text { Observational } \\
\text { Prospective }\end{array}$ & & 40 & & $\mathrm{x}$ & & & & $\mathrm{x}$ \\
\hline $\begin{array}{l}\text { Winkelmüller } \\
\text { \& Winkelmüller } \\
1996 \text { (79) }\end{array}$ & $\begin{array}{l}\text { Observational } \\
\text { Retrospective }\end{array}$ & 53 & 120 & & & & $\mathrm{x}$ & & $\mathrm{x}$ \\
\hline
\end{tabular}

nal catheter challenges) (80). Onofrio and Yaksh (82) investigated characteristics of intrathecal morphine analgesia in terminal metastatic disease. The median post-implant survival time was 4 months. Morphine was exclusively used for the trial and the infusion. An analgesic index was calculated at trial time and consisted of duration of pain relief in hours $x$ magnitude of relief/ morphine dose in $\mathrm{mg}$. Endpoints of the study included parenteral narcotic use, analgesic index, mobility, and overall outcome and prognostic value of the trial to infusion success. At 16 weeks post infusion, the average dose of intrathecal morphine was $9.5 \pm 2.1 \mathrm{mg} / \mathrm{d}$ compared to $3.7 \pm 0.3 \mathrm{mg} / \mathrm{d}$ at 2 weeks. Sixty-five percent of 26 patients with infusions that exceeded 26 weeks selfreported good or excellent results, while all 53 patients had improved analgesic index and mobility improved. Furthermore, patients that with a low analgesic index at trial were noted to require rapid dose escalation and that correlated with poor overall outcome, suggesting utility of the index in a standard trialing method. Miscalculation of pump refill dates led to severe pain in 5 cases (82). Penn and Paice (81) investigated pain relief by self-reported NRS, enteral opioid consumption, and self-reported daily activity logs in 43 patients, 35 of whom had intractable cancer pain. Patients were asked to categorize pain as excellent (0-3 on 0-10 NRS, greater than $50 \%$ reduction of oral narcotics, with significant increase in daily activities), good (4-6 on NRS, less than $50 \%$ reduction in oral narcotics, and some improvement in activities) to poor and failure. Seventeen of 35 cancer patients had excellent results based on the above criteria, while $80 \%$ had good or excellent results. No complications were attributed to the hardware or the infusion (81). Summary results of cancer-related pain studies are displayed in Table 11.

Systematic reviews on intrathecal drug delivery for 
pain have been performed by several groups $(24,29,98$ 101), including organizations that attempt to make recommendations in regard to reimbursement/coverage (98). The methodologies utilized varied significantly. On one end of the spectrum, case reports were allowed, and on the other end, only long-term assessments with specific documentation of indications and medications utilized. All of the reviews recognize that there is a paucity of good quality publications for intrathecal infusion therapy, especially for chronic, non-cancer pain. While the literature has significant heterogeneity of patient types, medications, and devices, there appears to effectiveness in regard to pain relief. The details of systematic reviews on intrathecal therapy for pain are detailed in Table 12. However, there is insufficient information for adequate comparison to other routes of

Table 11. Summary results of eligible cancer pain studies included in this systematic review.

\begin{tabular}{|c|c|c|c|c|c|c|c|}
\hline \multirow{2}{*}{ Study } & \multirow{2}{*}{ Study Characteristics } & \multirow{2}{*}{$\begin{array}{l}\text { Methodological Quality } \\
\text { Scoring }\end{array}$} & \multirow{2}{*}{$\begin{array}{l}\text { Number of } \\
\text { Participants }\end{array}$} & \multicolumn{2}{|c|}{$\geq 30 \%$ Pain Relief } & \multicolumn{2}{|c|}{$\geq 50 \%$ Pain Relief } \\
\hline & & & & 3 mos. & $\geq 6$ mos. & 3 mos. & $\geq 6 \mathrm{mos}$. \\
\hline Rauck et al, 2003 (74) & $\begin{array}{l}\text { Observational } \\
\text { Prospective }\end{array}$ & 60 & 149 & $\mathrm{x}$ & $\mathrm{x}$ & & \\
\hline Smith et al, 2002 (83) & $\begin{array}{c}\text { Randomized } \\
\text { Controlled Trial } \\
\end{array}$ & 64 & 202 & & & $\mathrm{x}$ & $\mathrm{x}$ \\
\hline Becker et al, 2000 (80) & $\begin{array}{l}\text { Observational } \\
\text { Retrospective }\end{array}$ & 53 & 43 & $\mathrm{x}$ & NA & $\mathrm{x}$ & NA \\
\hline Onofrio and Yaksh, 1990 (82) & $\begin{array}{l}\text { Observational } \\
\text { Prospective }\end{array}$ & 62 & 53 & & NA & $\mathrm{x}$ & NA \\
\hline Penn and Paice, 1987 (81) & $\begin{array}{l}\text { Observational } \\
\text { Retrospective }\end{array}$ & 60 & 43 & $\mathrm{x}$ & NA & $\mathrm{x}$ & NA \\
\hline
\end{tabular}

Table 12. Summary description of systematic reviews for intrathecal drug delivery.

\begin{tabular}{|c|c|c|c|c|c|c|c|}
\hline $\begin{array}{c}\text { Systematic } \\
\text { Review }\end{array}$ & Inclusion Criteria & Exclusion Criteria & $\begin{array}{l}\text { Number } \\
\text { of Studies/ } \\
\text { Patients }\end{array}$ & Outcome Measures & Outcomes & Complications & Conclusions \\
\hline $\begin{array}{l}\text { Patel et al, } \\
2009(24)\end{array}$ & $\begin{array}{l}\text { Fixed rate or } \\
\text { programmable } \\
\text { intrathecal infusion } \\
\text { pump studies for } \\
\text { chronic non-cancer } \\
\text { pain. Specific } \\
\text { indication for } \\
\text { intrathecal infusion } \\
\text { and drug utilized } \\
\text { Minimum } 12 \\
\text { months duration } \\
\text { Clear documentation } \\
\text { of outcomes and } \\
\text { complications } \\
\text { Minimum patient } \\
\text { number = 25 }\end{array}$ & $\begin{array}{l}\text { Lack of clear } \\
\text { documentation } \\
\text { of infusion } \\
\text { systems or mixed } \\
\text { delivery methods } \\
\text { Externalized } \\
\text { infusion systems } \\
\text { for short-term use } \\
\text { Less than } 12 \\
\text { months follow-up } \\
\text { Lack of clear } \\
\text { documentation } \\
\text { of indications and } \\
\text { patient population } \\
\text { being studied }\end{array}$ & $\begin{array}{l}4 \\
\text { observational } \\
\text { studies, } 386 \\
\text { patients }\end{array}$ & $\begin{array}{l}\text { Primary outcome: > } \\
50 \% \text { relief } \\
\text { Secondary } \\
\text { outcomes: } \\
\text { improvement of } \\
\text { function, reduction } \\
\text { in amount of } \\
\text { oral medication, } \\
\text { decrease in side } \\
\text { effects from } \\
\text { systemic drugs, } \\
\text { improvement in } \\
\text { quality of life }\end{array}$ & $\begin{array}{l}2 \text { studies were positive } \\
\text { for efficacy, one was } \\
\text { negative, one study } \\
\text { was not applicable }\end{array}$ & Not assessed & $\begin{array}{l}\text { Intrathecal } \\
\text { infusion devices } \\
\text { for chronic } \\
\text { non-cancer pain } \\
\text { have positive long- } \\
\text { term outcomes } \\
\text { and a role as an } \\
\text { advanced-stage } \\
\text { therapy, based on } \\
\text { limited studies }\end{array}$ \\
\hline $\begin{array}{l}\text { Noble et al, } \\
2008 \text { (98) }\end{array}$ & $\begin{array}{l}\text { English language } \\
\text { Controlled trials } \\
\text { and uncontrolled } \\
\text { long-term case } \\
\text { series. Prospective } \\
\text { and retrospective. } \\
\text { Chronic non-cancer } \\
\text { pain. Minimum } \\
\text { of } 10 \text { implanted } \\
\text { infusion pump } \\
\text { patients } \\
\text { Minimum of } 6 \\
\text { months for efficacy } \\
\text { data }\end{array}$ & $\begin{array}{l}\text { Outcomes } \\
\text { requiring patients } \\
\text { to remember their } \\
\text { previous health }\end{array}$ & $\begin{array}{l}16 \text { studies, } \\
2081 \text { patients }\end{array}$ & $\begin{array}{l}25 \% \text { and } 50 \% \\
\text { pain relief. } \\
\text { Discontinuation } \\
\text { from trial due to } \\
\text { insufficient pain } \\
\text { relief. Quality of Life } \\
\text { (Tollison, SF-36, } \\
\text { and QUALEFFO). } \\
\text { Functional status. } \\
\text { Employment } \\
\text { status. Use of other } \\
\text { medications or } \\
\text { treatments. Change } \\
\text { in medication } \\
\text { over time. } \\
\text { Discontinuation due } \\
\text { to AEs. MAUDE } \\
\text { reports. Cost }\end{array}$ & $\begin{array}{l}\text { 25\% relief: } 56.3 \% \text {. } \\
50 \% \text { relief: } 40.8 \% \text {. } \\
\text { Inadequate relief } \\
\text { discontinuation: } \\
8 \% \text {. Quality of life: } \\
\text { inconsistent findings. } \\
\text { Functional status and } \\
\text { Employment status: } \\
\text { insufficient quantity of } \\
\text { evidence. Use of other } \\
\text { medications: Overall } \\
\text { decrease. Dose of } \\
\text { medication increased } \\
\text { over time but quantity } \\
\text { is not predictable. } \\
\text { Discontinuation from } \\
\text { AEs: } 8.3 \%\end{array}$ & $\begin{array}{l}\text { Reoperation: } \\
\text { 9\%-42\%. } \\
\text { PDPH 0\%- } \\
\text { 31\%. Pump } \\
\text { failure: } 0 \%-8 \% \\
\text { Seroma: } 0 \%- \\
\text { 6\%. No serious } \\
\text { drug related } \\
\text { adverse events }\end{array}$ & $\begin{array}{l}\text { Effectiveness } \\
\text { data of IT pumps } \\
\text { for chronic } \\
\text { non-cancer } \\
\text { pain only from } \\
\text { uncontrolled case } \\
\text { series. Inconsistent } \\
\text { findings among } \\
\text { studies for } \\
\text { pain relief. } \\
\text { Inadequate data } \\
\text { for conclusions of } \\
\text { QOL or functional } \\
\text { status. Adverse } \\
\text { events included } \\
\text { reoperation } \\
\text { but difficult to } \\
\text { conclude mean } \\
\text { incidence. }\end{array}$ \\
\hline
\end{tabular}


Table 12 (cont.). Summary description of systematic reviews for intrathecal drug delivery.

\begin{tabular}{|c|c|c|c|c|c|c|c|}
\hline $\begin{array}{c}\text { Systematic } \\
\text { Review }\end{array}$ & Inclusion Criteria & Exclusion Criteria & $\begin{array}{l}\text { Number } \\
\text { of Studies/ } \\
\text { Patients }\end{array}$ & Outcome Measures & Outcomes & Complications & Conclusions \\
\hline $\begin{array}{l}\text { Turner et } \\
\text { al, } 2007 \\
(29)\end{array}$ & $\begin{array}{l}\text { 1) English language } \\
\text { journal article, } \\
\text { 2) Intrathecal opioid } \\
\text { or ziconotide via } \\
\text { programmable } \\
\text { pump, } \\
\text { 3) Patient diagnoses } \\
\text { not limited to } \\
\text { spasticity or specific } \\
\text { diseases } \\
\text { 4) Original data on } \\
\text { pain, functioning, } \\
\text { or complications in } \\
\text { humans }\end{array}$ & $\begin{array}{l}\text { 1) Constant } \\
\text { flow pumps or } \\
\text { unknown type of } \\
\text { pump } \\
\text { 2) }>10 \% \text { were } \\
\text { treated for } \\
\text { spasticity or a } \\
\text { specific disease } \\
\text { 3) Study only } \\
\text { focused on } \\
\text { patients who failed } \\
\text { the first IT drug } \\
\text { received } \\
\text { 4) Case reports }\end{array}$ & $\begin{array}{l}6 \\
\text { observational } \\
\text { articles met } \\
\text { criteria for } \\
\text { effectiveness } \\
\text { and } \\
\text { complication } \\
\text { review, } \\
\text { additional } 4 \\
\text { met criteria for } \\
\text { complication } \\
\text { review only } \\
\text { Effectiveness: } \\
342 \text { trialed, } \\
258 \text { implanted } \\
\text { Complications: } \\
377 \text { trialed, } \\
342 \text { implanted }\end{array}$ & $\begin{array}{l}\text { Effectiveness: pain } \\
\text { (VAS and NRS) and } \\
\text { functioning (CIPI } \\
\text { and Oswestry) } \\
\text { Complications: } \\
\text { a- biologic, b- } \\
\text { hardware related } \\
\text { Follow up was over } \\
\text { 3-60 months }\end{array}$ & $\begin{array}{l}\text { Pain improved on } \\
\text { average across all } \\
\text { studies, but high } \\
\text { attrition rate, and } \\
\text { increased opioid } \\
\text { consumption over } \\
\text { time. } \\
\text { All had some } \\
\text { improvement in } \\
\text { functioning but all had } \\
\text { methodology flaws. }\end{array}$ & $\begin{array}{l}\text { Wound } \\
\text { infection- } 12 \% \text {, } \\
\text { meningitis- } \\
2 \%, \text { CSF leak } \\
-0 \%, \text { N/V- } \\
33 \% \text {, Sedation- } \\
2 \% \text {, urinary } \\
\text { retention- } \\
24 \% \text {, } \\
\text { pruritus- } 26 \% \text {, } \\
\text { respiratory } \\
\text { depression- } \\
0 \% \text {, sexual } \\
\text { dysfunction- } \\
25 \% \text {, } \\
\text { constipation- } \\
38 \% \text {, catheter } \\
\text { related } \\
\text { complication } \\
-18 \% \text {, pump } \\
\text { failure- } 5 \% \text {, } \\
\text { reoperation- } \\
27 \% \text {, pump } \\
\text { explantation- } \\
5 \%\end{array}$ & $\begin{array}{l}\text { IT therapy } \\
\text { improves pain } \\
\text { scores, but } \\
\text { increases in } \\
\text { medication } \\
\text { consumption } \\
\text { occur, long-term } \\
\text { effectiveness was } \\
\text { unclear. Adverse } \\
\text { effects were } \\
\text { common and } \\
\text { often transient. }\end{array}$ \\
\hline $\begin{array}{l}\text { Waara- } \\
\text { Wolleat } \\
\text { et al, } 2006 \\
(100)\end{array}$ & $\begin{array}{l}\text { Medline search } \\
\text { and non-indexed } \\
\text { publications } \\
\text { (Neuromodulation) } \\
\text { Intrathecal fentanyl } \\
\text { or sufentanil }\end{array}$ & $\begin{array}{l}\text { 1) Acute clinical } \\
\text { use such as } \\
\text { preoperative } \\
\text { analgesia or for } \\
\text { labor }\end{array}$ & $\begin{array}{l}\text { Fentanyl: } 2 \\
\text { retrospective } \\
\text { analyses, total } \\
\mathrm{N}=36,1 \\
\text { prospective } \\
\text { study, } \mathrm{N}=69 \\
\text { Sufentanil: } 1 \\
\text { retrospective: } \\
\mathrm{N}=18\end{array}$ & $\mathrm{~N} / \mathrm{A}$ & $\begin{array}{l}\text { Fentanyl and } \\
\text { sufentanil were } \\
\text { efficacious and } \\
\text { tolerated in the } \\
\text { majority of patients }\end{array}$ & $\begin{array}{l}\text { Sufentanil } \\
\text { did not } \\
\text { produce lower } \\
\text { extremity } \\
\text { edema as } \\
\text { opposed to } \\
\text { morphine }\end{array}$ & $\begin{array}{l}\text { Limited number } \\
\text { of studies with } \\
\text { small number of } \\
\text { subjects suggest } \\
\text { that intrathecal } \\
\text { infusion of } \\
\text { lipophilic opioids } \\
\text { is generally } \\
\text { effective and well } \\
\text { tolerated, but } \\
\text { more studies are } \\
\text { recommended. }\end{array}$ \\
\hline $\begin{array}{l}\text { Simpson } \\
\text { et al, } 2003 \\
(99)\end{array}$ & $\begin{array}{l}\text { Intrathecal studies } \\
\text { Controlled studies } \\
\text { and case series } \\
\text { included }\end{array}$ & $\begin{array}{l}\text { If duplication } \\
\text { of patient } \\
\text { data, smaller } \\
\text { publication } \\
\text { excluded } \\
\text { RCTs comparing } \\
\text { baclofen versus } \\
\text { saline }\end{array}$ & $\begin{array}{l}1 \mathrm{RCT}, 6 \text { case } \\
\text { series, } 3 \text { cost } \\
\text { studies }\end{array}$ & $\begin{array}{l}\text { Pain reduction, } \\
\text { composite toxicity } \\
\text { score, safety, cost }\end{array}$ & $\begin{array}{l}\text { VAS } 1.97 \text { points less } \\
\text { than CMM patients } \\
\text { Composite toxicity } \\
\text { score } 2.82 \text { points better } \\
\text { than CMM } \\
\text { Baclofen had } \\
\text { statistically significant } \\
\text { improvement in } \\
\text { Ashworth score } \\
\text { Cost difficult to } \\
\text { calculate }\end{array}$ & $\begin{array}{l}\text { Surgical } \\
\text { revision: } \\
3-17 \% \\
\text { Pump } \\
\text { explantation: } \\
0-21 \% \\
\text { Catheter } \\
\text { complications: } \\
3-10 \% \\
\text { Dehiscence: } \\
2 \% \\
\text { Pocket erosion, } \\
\text { infection, } \\
\text { or seroma: } \\
2.4-4 \%\end{array}$ & $\begin{array}{l}\text { Intrathecal } \\
\text { infusion for pain } \\
\text { and spasticity } \\
\text { appears effective } \\
\text { for prescreened } \\
\text { patients. Therapy } \\
\text { appears safe, but } \\
\text { drug and device } \\
\text { complications } \\
\text { do occur and } \\
\text { may result in } \\
\text { surgical revision. } \\
\text { Intrathecal therapy } \\
\text { may be less costly } \\
\text { than medical } \\
\text { management in } \\
\text { the long-term. }\end{array}$ \\
\hline
\end{tabular}

delivery. In addition, there is significant lack of data in regard to the multitude of combinations possible between hydrophilic and lipophilic opioids, local anesthetics, clonidine, baclofen, and ziconotide. In regard to safety, drug related adverse effects are mostly transient and well tolerated. Device related complications and need for surgical revision appears to have occurred at a higher rate in earlier publications, and the lower rates in more recent publications may reflect improved education, techniques, and experience in the implanting community. Assessments of cost-effectiveness suggest that cost savings are achieved after 2 years in comparison to systemic pharmacologic therapy for chronic, non-cancer pain (101). 
Intrathecal Therapies for Cancer and Non-Cancer Pain

Table 12 (cont.). Summary description of systematic reviews for intrathecal drug delivery.

\begin{tabular}{|c|c|c|c|c|c|c|c|}
\hline $\begin{array}{c}\text { Systematic } \\
\text { Review }\end{array}$ & Inclusion Criteria & Exclusion Criteria & $\begin{array}{l}\text { Number } \\
\text { of Studies/ } \\
\text { Patients }\end{array}$ & Outcome Measures & Outcomes & Complications & Conclusions \\
\hline $\begin{array}{l}\text { Williams } \\
\text { et al, } 2000 \\
(101)\end{array}$ & $\begin{array}{l}\text { Search of Medline, } \\
\text { Embase, CancerCD, } \\
\text { and PubMed } \\
\text { Chronic cancer and } \\
\text { non-cancer pain in a } \\
\text { hospital, hospice, or } \\
\text { community setting } \\
\text { Different types of } \\
\text { intrathecal pump } \\
\text { systems } \\
\text { Different types of } \\
\text { intrathecal drugs } \\
\text { given by pump } \\
\text { systems } \\
\text { Comparison of } \\
\text { intrathecal delivery } \\
\text { systems with other } \\
\text { routes of analgesia } \\
\text { delivery } \\
\text { Case series and case } \\
\text { reports }\end{array}$ & $\begin{array}{l}\text { Publications that } \\
\text { did not specifically } \\
\text { measure efficacy } \\
\text { Review articles } \\
\text { with no original } \\
\text { information } \\
\text { Studies assessing } \\
\text { effectiveness of } \\
\text { epidural therapy } \\
\text { only } \\
\text { Some case series } \\
\text { reports excluded } \\
\text { for insufficient } \\
\text { information } \\
\text { of effects and } \\
\text { side-effects }\end{array}$ & $\begin{array}{l}49 \text { reports } \\
2,571 \text { patients }\end{array}$ & $\begin{array}{l}\text { 1) efficacy measures } \\
\text { included: VAS } \\
\text { Verbal Rating Score, } \\
\text { MPQ, Brief Pain } \\
\text { Inventory, range of } \\
\text { movement, ability to } \\
\text { return to work } \\
\text { 2) side-effects: (a) } \\
\text { pharmacological } \\
\text { side-effects } \\
\text { (e.g. respiratory } \\
\text { depression, effects } \\
\text { on motor and/ } \\
\text { or autonomic } \\
\text { function, nausea } \\
\text { and/or vomiting, } \\
\text { urinary retention, } \\
\text { pruritus); and (b) } \\
\text { complications (e.g. } \\
\text { local infection, } \\
\text { abscess formation, } \\
\text { meningitis, } \\
\text { bleeding/ hematoma } \\
\text { formation, pump } \\
\text { pocket seroma, } \\
\text { CSF leaks, dural } \\
\text { fistula, improper } \\
\text { pocket placement, } \\
\text { catheter kinking, } \\
\text { obstruction, } \\
\text { dislodgement, } \\
\text { disconnection, } \\
\text { malfunction, and } \\
\text { pump failure) } \\
\text { 3) costs: (a) costs } \\
\text { of IDDS, including } \\
\text { initial costs, } \\
\text { maintenance, } \\
\text { number of } \\
\text { outpatient visits, } \\
\text { hospital admissions } \\
\text { and use of health } \\
\text { care resources; } \\
\text { and (b) financial } \\
\text { benefits of the pump } \\
\text { systems, such as } \\
\text { reduction in drug } \\
\text { costs, reduction in } \\
\text { bed days, quicker } \\
\text { return to work, } \\
\text { reduction in the use } \\
\text { of health service } \\
\text { resources (GP visits, } \\
\text { outpatient visits) }\end{array}$ & $\begin{array}{l}\text { 1) Efficacy: mean } \\
\text { VAS pre-therapy was } \\
7.6 \text { and decreased to } \\
3.0 \text { with IT therapy } \\
\text { (16 studies), up } \\
\text { to } 50 \% \text { reduction } \\
\text { in supplemental } \\
\text { analgesia use, } 82.5 \% \\
\text { good to excellent } \\
\text { improvement in } \\
\text { daily activities, } \\
\text { improvement of } \\
\text { depression, } 77-92 \% \\
\text { patient satisfaction }\end{array}$ & $\begin{array}{l}\text { Nausea and } \\
\text { vomiting: 25\% } \\
\text { Sedation: 17\% } \\
\text { Urinary } \\
\text { retention: 19\% } \\
\text { Pruritus: 17\% } \\
\text { Myoclonic } \\
\text { activity: 18\% } \\
\text { Respiratory } \\
\text { depression: 3\% } \\
\text { Meningitis: 3\% } \\
\text { Catheter } \\
\text { dislodgment: } \\
\text { 5-18\% } \\
\text { Catheter } \\
\text { obstruction, } \\
\text { kinking } \\
\text { Pump failure } \\
\text { (battery, rotor } \\
\text { stall) } \\
\text { CSF leak, } \\
\text { seroma }\end{array}$ & $\begin{array}{l}\text { Quality of } \\
\text { literature is poor. } \\
\text { The heterogeneity } \\
\text { of medications, } \\
\text { devices, } \\
\text { and patient } \\
\text { populations make } \\
\text { firm conclusions } \\
\text { difficult. Efficacy } \\
\text { is documented, } \\
\text { but insufficient } \\
\text { information for } \\
\text { comparison to } \\
\text { other routes of } \\
\text { delivery. } \\
\text { Cost information } \\
\text { was difficult } \\
\text { to assess, but } \\
\text { modeling suggests } \\
\text { that IT therapy } \\
\text { is cost effective } \\
\text { after } 22 \text { months } \\
\text { in comparison to } \\
\text { systemic analgesic } \\
\text { therapy } \\
\text { Pharmacologic } \\
\text { adverse effects } \\
\text { occur in } 3-26 \% \\
\text { and mechanical } \\
\text { complications are } \\
\text { reported in up } \\
\text { to } 25 \%\end{array}$ \\
\hline
\end{tabular}

NAS = Numeric Analog Scale; VAS = Visual Analog Scale; NRS= Numeric Rating Scale; IT $=$ Intrathecal, IDDS = Intrathecal Drug Delivery System; CSF = Cerebrospinal Fluid; AE = Adverse Events, QUALEFFO = Quality of Life Questionnaire of the European Foundation for Osteoporosis; $\mathrm{QOL}=$ Quality of Life; MAUDE = Manufacturer and User Facility Device Experience Database; PDPH= Post-Dural Puncture Headache; CIPI= Chronic Illness Problem Inventory; N/V = Nausea/Vomiting; RCT = Randomized Controlled Trial; N/A = Not Applicable; MPQ = McGill Pain Questionnaire

\section{Level of Evidence}

The evidence for intrathecal infusion systems for non-cancer pain is Level II-3 based on USPSTF criteria.
There is Level II-2 evidence for intrathecal infusion systems for cancer-related pain. 


\section{Recommendation}

Based on Guyatt et al's criteria (66), the recommendation for intrathecal infusion systems is limited to moderate recommendation for non-cancer pain based on the current moderate evidence derived from randomized and observational studies for chronic c noncancer pain. The recommendation for cancer-related pain is moderate recommendation based on one high quality randomized controlled trial and evidence from from lesser quality studies.

\section{Discussion}

This systematic review presented evidence on the efficacy of IDDS in the control of cancer and non-cancer related pain. Based on the reviewed evidence, intrathecal therapy is moderately effective and safe in controlling refractory painful conditions that have failed multiple other treatment modalities, both in cancer and non-cancer related conditions. However, there are significant limitations to these inferences.

Significant variability in study design, patient selection, concomitant oral or transdermal opioid use and technical parameters may have important effects on outcomes of intrathecal therapies. Merely having an IDDS implanted does not impart similarity among patients' characteristics or response to therapy. Differences in patient selection, catheter location, medications used, complication rate, and location/type of pain treated may greatly affect outcomes and responses to therapy. Pharmacokinetic characteristics of intrathecally administered medications, particularly lipid solubility, play an important role in analgesic responsiveness. Consequently, the positioning of the catheter can be critical especially with use of lipophilic medications. Most intrathecal agents work by binding to particular receptors in the superficial layers of the dorsal horn. Prior to reaching their targets, intrathecal medications may be taken up by fat tissue and blood vessels. Lipophilic agents are more likely to be taken up by the systemic circulation than hydrophilic agents, as they diffuse easier across cell layers. Hence, hydrophilic opioids such as morphine and hydromorphone are sometimes preferred over hydrophobic opioids as they can diffuse in the CSF and have a higher chance of reaching target areas in the superficial layers of the dorsal horn that may not be immediately adjacent to the catheter tip (102). Unlike other agents used intrathecally, local anesthetics act earlier on sodium channels at the rootlets of nerve fibers (fila radicularia) in the intrathecal space; preferentially over targeting spinal cord receptors (103). Bupivacaine is the predominant local anesthetic used in chronic intrathecal infusion systems and is highly lipophilic. Peripherally generated painful stimuli are conducted along dorsal rootlets entering the dorsal horn of the spinal cord and not ventral rootlets. Hence, a dorsal location of the intrathecal catheter may be advantageous, albeit the dynamics of CSF flow in long-term intrathecal infusions are still undetermined $(102,103)$. None of the studies on intrathecal drug delivery addressed catheter location and only a few have looked at the efficacy of combination of intrathecal medications $(13,73,90,104-110)$. While preservative-free morphine and ziconotide are FDA-approved for the for intrathecal administration for the treatment of chronic pain, a number of other agents including bupivacaine, clonidine, and fentanyl are used often in combination with other agents. The lack of FDA approval for these medications hinders prospective studies and limits the potential to adequately investigate the effectiveness of these agents when used intrathecally, alone or in combination with other agents. Additionally, FDA approval for morphine and ziconotide is for monotherapy whereas combination therapy is often used in clinical practice. Combination therapy may prove to be superior to monotherapy given the complexity of pain signaling mechanisms; however, no human studies clearly attest to that potential. For instance, while neuraxial administration of a combination of local anesthetics and opioids is synergistic for pain relief in rats $(111,112)$, such assertion could not be easily made in human studies and may involve a number of variables $(73,102-104,110,113-117)$. A randomized double blind cross over study looking at the addition of bupivacaine to deliver 4,6 , or $8 \mathrm{mg} /$ day to an intrathecal pump already delivering chronic morphine or hydromorphone found no added benefit for bupivacaine (110). On the other hand, a double blind study of 20 cancer pain patients who have failed conservative medical management found that the combination of intrathecal morphine and bupivacaine blunted the escalation of intrathecal morphine dosing significantly (104). Intrathecal boluses of bupivacaine have been reported to control breakthrough pain in cancer pain patients within 10-15 minutes of administration $(114,118)$. Given the high lipid solubility of bupivacaine, catheter tip location is likely critical for its effectiveness in regional pain conditions (119). Of importance, the ability to treat breakthrough pain with intrathecal boluses through programmable pumps 
(Patient Therapy Manager®, Medtronic) has provided patients with substantial potential for improved relief (21). This relief may be experienced almost instantaneously when bupivacaine is administered and the catheter tip overlies the nerve(s) involved in transmission of the pain signal $(114,118)$; as bupivacaine works by blocking sodium channels predominantly on small nerve rootlets entering the spinal cord and less so by diffusing into the dorsal horn of the spinal cord (103). Whether the use of intrathecal bolus therapy increases efficacy of IDDS in managing chronic pain is unknown given the lack of such studies, especially in the setting of combination therapy, likely due to the above cited limitations.

Complications related to intrathecal therapy can be technical, biological, or medication related. While the vast majority of complications are minor, some serious complications can occur. An increased mortality rate in patients with non-cancer pain receiving intrathecal opioid therapy (mortality rate of $0.088 \%$ at 3 days after implantation, $0.39 \%$ at one-month, and $3.89 \%$ at oneyear) was identified as likely related to the opioids as well as other factors that may be mitigated especially at the start of therapy $(120,121)$. Other serious complications include granuloma formation that may be related to the amount and concentration of opiates, mostly morphine and hydromorphone $(47,122-127)$. Surgical interventions in these cases are rare (128) as most cases improve with weaning off the intrathecal opiate, replacing it with preservative-free saline which has been shown to reverse the course leading to resolution of the granuloma $(122,123)$. Granulomas may occur in as many as 3\% of implanted patients and most are asymptomatic (129). Routine MRIs to rule out intrathecal granulomas was not recommended by the authors of this prospective study given the relatively low incidence (129). The earliest sign of granuloma may be increased pain despite increasing opiate infusion; hence, clinical vigilance is of prime importance. Other complications of IDDS include catheter kinking, catheter fracture/ leakage, catheter migration, CSF leak, seroma, hygroma, infection, pump erosion through the skin, and medication side effects including but not limited to pruritus, nausea, vomiting, respiratory depression, and cognitive side effects.

The major limitation of this systematic review is the limited evidence for intrathecal infusion systems that is derived from randomized trials. Randomized trials provide valuable evidence about treatments and other interventions. However, most of the research and clini- cal practice comes from observational studies $(130,131)$. Randomized trials work by first assuming there is no difference between a new and an old, or placebo treatment to prove the null hypothesis (132). Basically, it may be described that the standard RCTs are in fact set up to show that treatments do not work, rather than to demonstrate that treatments do work (132). Essentially the RCTs are designed to stop therapeutic bandwagons in their tracks and also practioners peddling worthless treatments to patients made vulnerable and desperate by their illness. However, most questions in medical research are investigated in observational studies $(130,131,133-137)$. Consequently, observational studies are more likely to provide an indication of daily medical practice (138). Thus, the proponents of observational studies describe that observational studies are just as effective as RCTs. However, from a methodological perspective, the 2 types of studies are considered complementary rather than opposing (137). Thus, in clinical practice specifically involving interventions and surgery, observational studies and RCTs can be viewed as expressions in the setting of modern clinical research of the steps of observational and experimentation that form the basis of scientific methodology. Guyatt and Drummond (139) in a description of the hierarchy of strength of evidence for treatment decisions provide significant strength to systematic reviews of observational studies and single observational studies. Further disadvantages have been noted about the observational studies where research is often not detailed and clear enough to assess the strength and weaknesses of the investigations $(130,131,140-158)$. However, in health technology assessment, Deeks et al (144) concluded that the results of observational studies sometimes, but not always, differ from results of randomized studies of the same intervention. Further, Hartz et al (159) in assessing observational studies of medical treatments, concluded that reporting was often inadequate to compare the study designs or allow other meaningful interpretation of results.

However, the concept that assignment of subjects randomly to either experimental or control groups as the perfect science has been questioned (160). While researchers believe that randomization ensures that participating groups will differ only by chance, it does not guarantee that the balance will actually be achieved through randomization (161-163). In fact, in a comparison of randomized and observational samples, there was only one significant difference when patients were allocated by means of non-randomization among 
groups or compared to the total sample, in contrast to randomization showing significant difference in 7 parameters indicating that a randomized design may not be the best in interventional pain management settings (162). Further, issue of placebo is extremely crucial and quite difficult in interventional pain management settings with treatments such as intrathecal infusion systems given ethical and legal issues with currently only two agents being FDA approved and having only a single chamber intrathecal pump. In contrast, Benson and Hartz (164) in a 2000 publication comparing observational studies and RCTs, found little evidence that estimates of treatment effects in observational studies reported after 1984, were either consistently larger than or qualitatively different from those obtained in RCTs. Hartz et al (165), in assessing observational studies of chemonucleolysis, concluded that the results suggested that review of several comparable observational studies may help evaluate treatment, identify patient types most likely to benefit from a given treatment, and provide information about study features that improve the design of subsequent observational studies or even RCTs. However, they also cautioned that potential of comparative observational studies has not been realized because of concurrent inadequacies in their design, analysis, and reporting. Concato et al (166) in a 2000 publication evaluating published articles in 5 major medical journals from 1991 to 1995 , concluded that the results of well-designed observational studies do not systematically overestimate the magnitude of the effects of treatment as compared with those in RCTs on the same topic. Shrier et al (167) found that the advantages of including both observational studies and randomized trials in a meta-analysis could outweigh the disadvantages in many situations and that observational studies should not be excluded a priori. Thus, we believe that this review provides optimal evaluation including randomized and observational studies as well as evaluating for chronic cancer and non-cancer pain.

Given all the above and despite the potential for serious complications, intrathecal drug delivery remains a valuable therapy for chronic painful conditions, both cancer and non-cancer related. This therapy is often employed as a last resort in patients who have failed multiple other treatment modalities and has been shown to be effective when other interventions have failed. Significant challenges limit progress with intrathecal therapies. These may include the complex nature of pain and the frequent need to use a combination of drugs, many of which are not FDA approved for use in the intrathecal space. The current use of a single-chamber pump to deliver multiple medications poses particular dosing challenges when combination therapy is employed. Additionally, poor reimbursement for what is deemed a labor intensive task that requires high level vigilance from the managing physician is limiting the access of deserving patients to this important form of therapy (168). This factor may additionally limit the development of needed novel agents that would offer improved analgesia and would have a more favorable safety profile. It is hoped that this therapy will remain a viable option for effective pain relief for patients with refractory chronic pain and that technological and pharmacological developments will improve its safety and efficacy in the future.

\section{Acknowledgments}

We would like to thank the editorial board of Pain Physician for review and criticism in improving th manuscript.

\section{Disclosures}

Author Contributions: Dr. Hayek had full access to all the data in the study and takes responsibility for the integrity of the data and the accuracy of the data analysis. Drs. Hayek, Deer, Pope and Patel designed the study protocol. Dr. Hayek managed the literature searches and summaries of previous related work and wrote the firsts draft of the manuscript. All other authors provided revision for intellectual content and final approval of the manuscript.

Conflict of Interest: All authors have no conflicts of interest to report relevant to intrathecal therapy. None of the authors of the manuscript received any remuneration for writing the manuscript. Further, the authors have not received any reimbursement or honorarium in any other manner. Deer is a consultant for Medtronic, Medasys, and Codman. The authors are not affiliated in any manner with manufacturers of pain pumps. However, all the authors are members of the American Society of Interventional Pain Physicians (ASIPP) and practicing interventional pain physicians.

Funding/Support: There was no financial support or sponsorship for the study or its expenses. 


\section{References}

1. Yaksh TL, Rudy TA. Analgesia mediated by a direct spinal action of narcotics. Science 1976; 192:1357-1358.

2. Atweh SF, Kuhar MJ. Autoradiographic localization of opiate receptors in rat brain. I. Spinal cord and lower medulla. Brain Res 1977; 124:53-67.

3. Belverud S, Mogilner A, Schulder M. Intrathecal pumps. Neurotherapeutics 2008; 5:114-122.

4. Krames ES. Intrathecal infusional therapies for intractable pain: Patient management guidelines. J Pain Symptom Manage 1993; 8:36-46.

5. Manchikanti L, Boswell MV, Singh $\mathrm{V}$, Benyamin RM, Fellows B, Abdi S, Buenaventura RM, Conn A, Datta S, Derby R, Falco FJE, Erhart S, Diwan S, Hayek SM, Helm S, Parr AT, Schultz DM, Smith HS, Wolfer LR, Hirsch JA. Comprehensive evidence-based guidelines for interventional techniques in the management of chronic spinal pain. Pain Physician 2009; 12:699-802.

6. Manchikanti L, Boswell MV, Datta S, Fellows B, Abdi S, Singh V, Benyamin RM, Falco FJE, Helm S, Hayek S, Smith HS. Comprehensive review of therapeutic interventions in managing chronic spinal pain. Pain Physician 2009; 12:E123E198.

7. Manchikanti L, Helm S, Singh V, Benyamin RM, Datta S, Hayek S, Fellows B, Boswell MV. An algorithmic approach for clinical management of chronic spinal pain. Pain Physician 2009; 12:E225E264.

8. Gupta A, Mehdi A, Duwell M, Sinha A. Evidence-based review of the pharmacoeconomics related to the management of chronic nonmalignant pain. J Pain Palliat Care Pharmacother 2010; 24:152-156.

9. Manchikanti L, Singh V, Datta S, Cohen SP, Hirsch JA. Comprehensive review of epidemiology, scope, and impact of spinal pain. Pain Physician 2009; 12:E35E7o.

10. Dagenais S, Caro J, Haldeman S. A systematic review of low back pain cost of illness studies in the United States and internationally. Spine J 2008; 8:8-20.

11. Deyo RA, Mirza SK, Turner JA, Martin BI. Overtreating chronic back pain: Time to back off? J Am Board Fam Med 2009; 22:62-68.

12. Peng PW, Fedoroff I, Jacques L, Kumar K. Survey of the practice of spinal cord stimulators and intrathecal analgesic delivery implants for management of pain in Canada. Pain Res Manag 2007; 12:281-285.

13. Deer TR, Kim C, Bowman R, Tolentino D, Stewart C, Tolentino W. Intrathecal ziconotide and opioid combination therapy for noncancer pain: An observational study. Pain Physician 2009; 12:E291E296.

14. Raffaeli W, Sarti D, Demartini L, Sotgiu A, Bonezzi C; Italian Ziconotide Group. Italian registry on long-term intrathecal ziconotide treatment. Pain Physician 2011; 14:15-24.

15. Lawson EF, Wallace MS. Current developments in intraspinal agents for cancer and noncancer pain. Curr Pain Headache Rep 2010; 14:8-16.

16. Bennett G, Burchiel K, Buchser E, Classen A, Deer T, Du Pen S, Ferrante FM, Hassenbusch SJ, Lou L, Maeyaert J, Penn R, Portenoy RK, Rauck R, Serafini M, Willis KD, Yaksh T. Clinical guidelines for intraspinal infusion: Report of an expert panel. PolyAnalgesic Consensus Conference 2000. J Pain Symptom Manage 2000; 20:S37-43.

17. Deer TR, Smith HS, Cousins M, Doleys DM, Levy RM, Rathmell JP, Staats PS, Wallace M, Webster LR. Consensus guidelines for the selection and implantation of patients with noncancer pain for intrathecal drug delivery. Pain Physician 2010; 13:E175-213.

18. Deer T, Krames ES, Hassenbusch SJ, Burton A, Caraway D, Dupen S, Eisenach J, Erdek M, Grigsby E, Kim P, Levy R, McDowell G, Mekhail N, Panchal S, Prager J, Rauck R, Saulino M, Sitzman $T$, Staats $P$, Stanton-Hicks $M$, Stearns L, Willis KD, Witt W, Follett K, Huntoon M, Liem L, Rathmell J, Wallace M, Buchser E, Cousins M, Ver Donck A. Polyanalgesic Consensus Conference 2007: Recommendations for the management of pain by intrathecal (intraspinal) drug delivery: Report of an interdisciplinary expert panel. Neuromodulation 2007; 10:300-328.

19. Hassenbusch SJ, Portenoy RK, Cousins $M$, Buchser $E$, Deer TR, Du Pen SL, Eisenach J, Follett KA, Hildebrand KR, Krames ES, Levy RM, Palmer PP, Rathmell JP, Rauck RL, Staats PS, Stearns L, Willis KD. Polyanalgesic Consensus Conference 2003: An update on the management of pain by intraspinal drug delivery-- report of an expert panel. J Pain Symptom Manage 2004; 27:540-
563.

20. Duse G, Davia G, White PF. Improvement in psychosocial outcomes in chronic pain patients receiving intrathecal morphine infusions. Anesth Analg 2009; 109:1981-1986.

21. Ilias W, le Polain B, Buchser E, Demartini L. Patient-controlled analgesia in chronic pain patients: Experience with a new device designed to be used with implanted programmable pumps. Pain Pract 2008; 8:164-170.

22. Deer T, Chapple I, Classen A, Javery K, Stoker V, Tonder L, Burchiel K. Intrathecal drug delivery for treatment of chronic low back pain: Report from the $\mathrm{Na}$ tional Outcomes Registry for Low Back Pain. Pain Med 2004; 5:6-13.

23. Dewilde S, Verdian L, Maclaine GD. Cost-effectiveness of ziconotide in intrathecal pain management for severe chronic pain patients in the UK. Curr Med Res Opin 2009; 25:2007-2019.

24. Patel VB, Manchikanti L, Singh V, Schultz DM, Hayek SM, Smith HS. Systematic review of intrathecal infusion systems for long-term management of chronic non-cancer pain. Pain Physician 2009; 12:345-360.

25. Kumar K, Hunter G, Demeria DD. Treatment of chronic pain by using intrathecal drug therapy compared with conventional pain therapies: A cost-effectiveness analysis. J Neurosurg 2002; 97:803810.

26. Hassenbusch SJ, Paice JA, Patt RB, Bedder MD, Bell GK. Clinical realities and economic considerations: Economics of intrathecal therapy. J Pain Symptom Manage 1997; 14:S36-S48.

27. de Lissovoy G, Brown RE, Halpern M, Hassenbusch SJ, Ross E. Cost-effectiveness of long-term intrathecal morphine therapy for pain associated with failed back surgery syndrome. Clin Ther 1997; 19:96-112.

28. Smith TJ, Coyne PJ. Implantable drug delivery systems (IDDS) after failure of comprehensive medical management (CMM) can palliate symptoms in the most refractory cancer pain patients. J Palliat Med 2005; 8:736-742.

29. Turner JA, Sears JM, Loeser JD. Programmable intrathecal opioid delivery systems for chronic non-malignant pain: A systematic review of effectiveness and complications. Clin ] Pain 2007; 23:180-195.

30. Noble M, Treadwell J, Schoelles K, Sun F. Health Technology Assessment, 
Washington State Health Care Authority. Implantable Infusion Pumps for Chronic Noncancer Pain. ECRI Institue, July 18, 2008.

www.hta.hca.wa.gov/documents/implantable_infusion_pumps_final.pdf

31. Farrar JT, Young JP, Jr., LaMoreaux L, Werth JL, Poole RM. Clinical importance of changes in chronic pain intensity measured on an 11-point numerical pain rating scale. Pain 2001; 94:149-158.

32. West S, King V, Carey TS, Lohr KN, McKoy N, Sutton SF, Lux L. Systems to Rate the Strength of Scientific Evidence. Evidence Report/Technology Assessment No. 47. AHRQ Publication No. 02-Eo16. Agency for Healthcare Research and Quality, Rockville, MD, 2002.

33. Manchikanti L, Singh V, Smith HS, Hirsch JA. Evidence-based medicine, systematic reviews, and guidelines in interventional pain management: Part 4: Observational studies. Pain Physician 2009; 12:73-108.

34. Manchikanti L, Datta S, Smith HS, Hirsch JA. Evidence-based medicine, systematic reviews, and guidelines in interventional pain management: Part 6: Systematic reviews and meta-analyses of observational studies. Pain Physician 2009; 12:819-850.

35. Koes BW, Scholten RJ, Mens JM, Bouter LM. Efficacy of epidural steroid injections for low-back pain and sciatica: A systematic review of randomized clinical trials. Pain 1995; 63:279-288.

36. Koes BW, Scholten RJ, Mens JMA, Bouter LM. Epidural steroid injections for low back pain and sciatica. An updated systematic review of randomized clinical trials. Pain Digest 1999; 9:241-247.

37. Nelemans $P$, de Bie R, de Vet $H$, Sturmans F. WITHDRAWN: Injection therapy for subacute and chronic benign lowback pain. Cochrane Database Syst Rev 2007; 3:CDoo1824.

38. Staal JB, de Bie RA, de Vet HC, Hildebrandt J, Nelemans $P$. Injection therapy for subacute and chronic low back pain: An updated Cochrane review. Spine (Phila Pa 1976) 2009; 34:49-59.

39. Staal JB, de Bie R, de Vet HC, Hildebrandt J, Nelemans P. Injection therapy for subacute and chronic low-back pain. Cochrane Database Syst Rev 2008; 3:CDool824.

40. Manchikanti L, Hirsch JA, Smith HS. Evidence-based medicine, systematic reviews, and guidelines in interventional pain management: Part 2: Randomized controlled trials. Pain Physician 2008;
11:717-773.

41. Manchikanti L, Benyamin RM, Helm S, Hirsch JA. Evidence-based medicine, systematic reviews, and guidelines in interventional pain management: Part 3: Systematic reviews and meta-analysis of randomized trials. Pain Physician 2009; 12:35-72.

42. Manchikanti L, Derby R, Wolfer LR, Singh V, Datta S, Hirsch JA. Evidencebased medicine, systematic reviews, and guidelines in interventional pain management: Part 5: Diagnostic accuracy studies. Pain Physician 2009; 12:517-540.

43. Manchikanti L, Datta S, Derby R, Wolfer LR, Benyamin RM, Hirsch JA. A critical review of the American Pain Society clinical practice guidelines for interventional techniques: Part 1. Diagnostic interventions. Pain Physician 2010; 13:E141E174.

44. Benyamin RM, Singh V, Parr AT, Conn A, Diwan S, Abdi S. Systematic review of the effectiveness of cervical epidurals in the management of chronic neck pain. Pain Physician 2009; 12:137-157.

45. Buenaventura RM, Datta S, Abdi S, Smith HS. Systematic review of therapeutic lumbar transforaminal epidural steroid injections. Pain Physician 2009; 12:233-251.

46. Conn A, Buenaventura RM, Datta S, Abdi S, Diwan S. Systematic review of caudal epidural injections in the management of chronic low back pain. Pain Physician 2009; 12:109-135.

47. Manchikanti L, Dunbar EE, Wargo BW, Shah RV, Derby R, Cohen SP. Systematic review of cervical discography as a diagnostic test for chronic spinal pain. Pain Physician 2009; 12:305-321.

48. Epter RS, Helm S, 2nd, Hayek SM, Benyamin RM, Smith HS, Abdi S. Systematic review of percutaneous adhesiolysis and management of chronic low back pain in post lumbar surgery syndrome. Pain Physician 2009; 12:361-378.

49. Frey ME, Manchikanti L, Benyamin RM, Schultz DM, Smith HS, Cohen SP. Spinal cord stimulation for patients with failed back surgery syndrome: A systematic review. Pain Physician 2009; 12:379397.

50. Gerges FJ, Lipsitz SR, Nedeljkovic SS. A systematic review on the effectiveness of the Nucleoplasty procedure for discogenic pain. Pain Physician 2010; 13:117132.

51. Hayek SM, Helm S, Benyamin RM, Singh V, Bryce DA, Smith HS. Effectiveness of spinal endoscopic adhesioly- sis in post lumbar surgery syndrome: A systematic review. Pain Physician 2009; 12:419-435.

52. Helm S, Hayek SM, Benyamin RM, Manchikanti L. Systematic review of the effectiveness of thermal annular procedures in treating discogenic low back pain. Pain Physician 2009; 12:207-232.

53. Manchikanti L, Derby R, Benyamin RM, Helm S, Hirsch JA. A systematic review of mechanical lumbar disc decompression with nucleoplasty. Pain Physician 2009; 12:561-572.

54. Manchikanti L, Glaser SE, Wolfer L, Derby R, Cohen SP. Systematic review of lumbar discography as a diagnostic test for chronic low back pain. Pain Physician 2009; 12:541-559.

55. Parr AT, Diwan S, Abdi S. Lumbar interlaminar epidural injections in managing chronic low back and lower extremity pain: A systematic review. Pain Physician 2009; 12:163-188.

56. Singh V, Benyamin RM, Datta S, Falco FJ, Helm S, 2nd, Manchikanti L. Systematic review of percutaneous lumbar mechanical disc decompression utilizing Dekompressor. Pain Physician 2009; 12:589-599.

57. Singh V, Manchikanti L, Benyamin RM, Helm S, Hirsch JA. Percutaneous lumbar laser disc decompression: A systematic review of current evidence. Pain Physician 2009; 12:573-588.

58. Falco FJE, Erhart S, Wargo BW, Bryce DA, Atluri S, Datta S, Hayek SM. Systematic review of diagnostic utility and therapeutic effectiveness of cervical facet joint interventions. Pain Physician 2009; 12:323-344.

59. Datta S, Lee M, Falco FJE, Bryce DA, Hayek SM. Systematic assessment of diagnostic accuracy and therapeutic utility of lumbar facet joint interventions. Pain Physician 2009; 12:437-460.

6o. Smith HS, Chopra P, Patel VB, Frey ME, Rastogi R. Systematic review on the role of sedation in diagnostic spinal interventional techniques. Pain Physician 2009; 12:195-206.

61. Rupert MP, Lee M, Manchikanti L, Datta S, Cohen SP. Evaluation of sacroiliac joint interventions: A systematic appraisal of the literature. Pain Physician 2009; 12:399-418.

62. Manchikanti L, Falco FJE, Boswell MV, Hirsch JA. Facts, fallacies, and politics of comparative effectiveness research: Part 1. Basic considerations. Pain Physician 2010; 13:E23-E54.

63. Manchikanti L, Falco FJE, Boswell MV, 
Hirsch JA. Facts, fallacies, and politics of comparative effectiveness research: Part 2. Implications for interventional pain management. Pain Physician 2010; 13:E55-E79.

64. Manchikanti L, Datta S, Gupta S, Munglani R, Bryce DA, Ward SP, Benyamin RM, Sharma ML, Helm II S, Fellows B, Hirsch JA. A critical review of the American Pain Society clinical practice guidelines for interventional techniques: Part 2. Therapeutic interventions. Pain Physician 2010; 13:E215-E264.

65. Harris RP, Helfand M, Woolf SH, Lohr KN, Mulrow CD, Teutsch SM, Atkins D; Methods Work Group, Third US Preventive Services Task Force. Current methods of the US Preventive Services Task Force. Am J Prevent Med 2001; 20:21-35.

66. Guyatt G, Gutterman D, Baumann MH, Addrizzo-Harris D, Hylek EM, Phillips B, Raskob G, Lewis SZ, Schunemann H. Grading strength of recommendations and quality of evidence in clinical guidelines: Report from an American College of Chest Physicians Task Force. Chest 2006; 129:174-181.

67. Anderson VC, Burchiel KJ. A prospective study of long-term intrathecal morphine in the management of chronic nonmalignant pain. Neurosurgery 1999; 44:289-300.

68. Atli A, Theodore BR, Turk DC, Loeser JD. Intrathecal opioid therapy for chronic nonmalignant pain: A retrospective cohort study with 3-year follow-up. Pain Med 2010; 11:1010-1016.

69. Deer TR, Caraway DL, Kim CK, Dempsey $C D$, Stewart CD, McNeil KF. Clinical experience with intrathecal bupivacaine in combination with opioid for the treatment of chronic pain related to failed back surgery syndrome and metastatic cancer pain of the spine. Spine J 2002; 2:274-278.

70. Doleys DM, Brown JL, Ness T. Multidimensional outcomes analysis of intrathecal, oral opioid, and behavioral-functional restoration therapy for failed back surgery syndrome: A retrospective study with 4 years follow-up. Neuromodulation 2006; 9:270-283.

71. Dominguez E, Sahinler B, Bassam D, Day M, Lou L, Racz G, Raj P. Predictive value of intrathecal narcotic trials for long-term therapy with implantable drug administration systems in chronic non-cancer pain patients. Pain Pract 2002; 2:315-325.

72. Ellis DJ, Dissanayake S, McGuire D,
Charapata SG, Staats PS, Wallace MS, Grove GW, Vercruysse P. Continuous intrathecal infusion of ziconotide for treatment of chronic malignant and nonmalignant pain over 12 months: A prospective, open-label study. Neuromodulation 2008; 11:40-49.

73. Rainov NG, Heidecke V, Burkert W. Long-term intrathecal infusion of drug combinations for chronic back and leg pain. J Pain Symptom Manage 2001; 22:862-871.

74. Rauck RL, Cherry D, Boyer MF, Kosek P, Dunn J, Alo K. Long-term intrathecal opioid therapy with a patient-activated, implanted delivery system for the treatment of refractory cancer pain. J Pain 2003; 4:441-447.

75. Roberts LJ, Finch PM, Goucke CR, Price LM. Outcome of intrathecal opioids in chronic non-cancer pain. Eur J Pain 2001; 5:353-361.

76. Shaladi A, Saltari MR, Piva B, Crestani F, Tartari S, Pinato P, Micheletto G, Dall'Ara R. Continuous intrathecal morphine infusion in patients with vertebral fractures due to osteoporosis. Clin J Pain 2007; 23:511-517.

77. Staats $P$, Whitworth $M$, Barakat $M$, Anderson W, Lilienfeld $S$. The use of implanted programmable infusion pumps in the management of nonmalignant, chronic low-back pain. Neuromodulation 2007; 10:376-380.

78. Thimineur MA, Kravitz E, Vodapally MS. Intrathecal opioid treatment for chronic non-malignant pain: A 3-year prospective study. Pain 2004; 109:242-249.

79. Winkelmuller $M$, Winkelmuller W. Long-term effects of continuous intrathecal opioid treatment in chronic pain of nonmalignant etiology. J Neurosurg 1996; 85:458-467.

8o. Becker R, Jakob D, Uhle El, Riegel T, Bertalanffy $H$. The significance of intrathecal opioid therapy for the treatment of neuropathic cancer pain conditions. Stereotact Funct Neurosurg 2000; 75:1626.

81. Penn RD, Paice JA. Chronic intrathecal morphine for intractable pain. J Neurosurg 1987; 67:182-186.

82. Onofrio BM, Yaksh TL. Long-term pain relief produced by intrathecal morphine infusion in 53 patients. J Neurosurg 1990; 72:200-209.

83. Smith TJ, Staats PS, Deer T, Stearns LJ, Rauck RL, Boortz-Marx RL, Buchser E, Catala E, Bryce DA, Coyne PJ, Pool GE; Implantable Drug Delivery Systems
Study Group. Randomized clinical trial of an implantable drug delivery system compared with comprehensive medical management for refractory cancer pain: Impact on pain, drug-related toxicity, and survival. J Clin Oncol 2002; 20:40404049.

84. Staats PS, Yearwood T, Charapata SG, Presley RW, Wallace MS, Byas-Smith M, Fisher R, Bryce DA, Mangieri EA, Luther RR, Mayo M, McGuire D, Ellia D. Intrathecal ziconotide in the treatment of refractory pain in patients with cancer or AIDS: A randomized controlled trial. JAMA 2004; 291:63-70.

85. Mercadante $S$, Intravaia G, Villari P, Ferrera P, Riina S, David F, Mangione S. Intrathecal treatment in cancer patients unresponsive to multiple trials of systemic opioids. Clin J Pain 2007; 23:793798.

86. Hassenbusch SJ, Pillay PK, Magdinec M, Currie K, Bay JW, Covington EC, Tomaszewski MZ. Constant infusion of morphine for intractable cancer pain using an implanted pump. J Neurosurg 1990; 73:405-409.

87. Pasutharnchat K, Tan KH, Abdul Hadi $\mathrm{M}$, Ho KY. Intrathecal analgesia in patients with cancer pain--an audit in a tertiary institution. Ann Acad Med Singapore 2009; 38:943-946.

88. Gestin Y, Vainio A, Pegurier AM. Longterm intrathecal infusion of morphine in the home care of patients with advanced cancer. Acta Anaesthesiol Scand 1997; 41:12-17.

89. Devulder J, Ghys L, Dhondt W, Rolly G. Spinal analgesia in terminal care: Risk versus benefit. J Pain Symptom Manage 1994; 9:75-81.

90. Van Dongen RT, Crul BJ, De Bock M. Long-term intrathecal infusion of morphine and morphine/bupivacaine mixtures in the treatment of cancer pain: A retrospective analysis of 51 cases. Pain 1993; 55:119-123.

91. Rauck RL, Wallace MS, Leong MS, Minehart $M$, Webster LR, Charapata SG, Abraham JE, Buffington DE, Ellis $\mathrm{D}$, Kartzinel R. A randomized, double-blind, placebo-controlled study of intrathecal ziconotide in adults with severe chronic pain. J Pain Symptom Manage 2006; 31:393-406.

92. Wallace MS, Charapata SG, Fisher R, Byas-Smith M, Staats PS, Mayo M, McGuire D, Ellis D. Intrathecal ziconotide in the treatment of chronic nonmalignant pain: A randomized, double-blind, 
placebo-controlled clinical trial. Neuromodulation 2006; 9:75-86.

93. Deer TR, Penn R, Kim CK, Bowman RG, Norris M, Stewart CD, Garten TG, Tolentino W, Khan Y. The use of continuous intrathecal infusion of octreotide in patients with chronic pain of noncancer origin: An evaluation of efficacy in a prospective double-blind fashion. Neuromodulation 2006, 9:284-289.

94. Paice JA, Penn RD, Kroin JS. Intrathecal octreotide for relief of intractable nonmalignant pain: 5-year experience with two cases. Neurosurgery 1996; 38:203207.

95. Hassenbusch SJ, Stanton-Hicks M, Covington EC, Walsh JG, Guthrey DS. Longterm intraspinal infusions of opioids in the treatment of neuropathic pain. J Pain Symptom Manage 1995; 10:527-543.

96. Angel IF, Gould HJ, Jr., Carey ME. Intrathecal morphine pump as a treatment option in chronic pain of nonmalignant origin. Surg Neurol 1998; 49:92-98.

97. Kumar K, Kelly M, Pirlot T. Continuous intrathecal morphine treatment for chronic pain of nonmalignant etiology: Long-term benefits and efficacy. Surg Neurol 2001; 55:79-86.

98. Noble M, Tregear SJ, Treadwell JR, Schoelles K. Long-term opioid therapy for chronic noncancer pain: A systematic review and meta-analysis of efficacy and safety. J Pain Symptom Manage 2008; 35:214-228.

99. Simpson B, Middleton P, Maddern G. The Royal Australasian College of Surgeons Implantable spinal infusion devices for chronic pain and spasticity: An accelerated systematic review. ASERNIP-S REPORT NO. 2003; 42, 1-50.

100. Waara-Wolleat KL, Hildebrand KR, Stewart GR. A review of intrathecal fentanyl and sufentanil for the treatment of chronic pain. Pain Med 2006; 7:251-259.

101. Williams JE, Louw G, Towlerton G. Intrathecal pumps for giving opioids in chronic pain: A systematic review. Health Technol Assess 2000; 4:iii-iv,1-65.

102. Bernards CM. Recent insights into the pharmacokinetics of spinal opioids and the relevance to opioid selection. Curr Opin Anaesthesiol 2004; 17:441-447.

103. Bernards CM. Cerebrospinal fluid and spinal cord distribution of baclofen and bupivacaine during slow intrathecal infusion in pigs. Anesthesiology 2006; 105:169-178

104. van Dongen RT, Crul BJ, van Egmond J. Intrathecal coadministration of bupivacaine diminishes morphine dose pro- gression during long-term intrathecal infusion in cancer patients. Clin J Pain 1999; 15:166-172.

105. Ackerman LL, Follett KA, Rosenquist RW. Long-term outcomes during treatment of chronic pain with intrathecal clonidine or clonidine/opioid combinations. J Pain Symptom Manage 2003; 26:668-677.

106. Siddall PJ, Molloy AR, Walker S, Mather LE, Rutkowski SB, Cousins MJ. The efficacy of intrathecal morphine and clonidine in the treatment of pain after spinal cord injury. Anesth Analg 2000; 91:14931498.

107. Webster LR, Fakata KL, Charapata S, Fisher R, MineHart M. Open-label, multicenter study of combined intrathecal morphine and ziconotide: Addition of morphine in patients receiving ziconotide for severe chronic pain. Pain Med 2008; 9:282-290.

108. Wallace MS, Kosek PS, Staats P, Fisher R, Schultz DM, Leong M. Phase II, openlabel, multicenter study of combined intrathecal morphine and ziconotide: Addition of ziconotide in patients receiving intrathecal morphine for severe chronic pain. Pain Med 2008; 9:271-281.

109. Wallace MS, Rauck RL, Deer T. Ziconotide combination intrathecal therapy: Rationale and evidence. Clin J Pain 2010; 26:635-644.

110. Mironer YE, Haasis JC, Chapple I, Brown C, Satterthwaite JR. Efficacy and safety of intrathecal opioid/bupivacaine mixture in chronic nonmalignant pain: A double blind, randomized, crossover, multicenter study by the National Forum of Independent Pain Clinicians (NFIPC). Neuromodulation 2002; 5:208213.

111. Kaneko M, Saito Y, Kirihara Y, Collins JG, Kosaka Y. Synergistic antinociceptive interaction after epidural coadministration of morphine and lidocaine in rats. Anesthesiology 1994; 80:137-150.

112. Saito $Y$, Kaneko M, Kirihara Y, Sakura S, Kosaka Y. Interaction of intrathecally infused morphine and lidocaine in rats (Part I): Synergistic antinociceptive effects. Anesthesiology 1998; 89:1455-1463.

113. Dahm P, Nitescu P, Appelgren L, Curelaru I. Efficacy and technical complications of long-term continuous intraspinal infusions of opioid and/or bupivacaine in refractory nonmalignant pain: A comparison between the epidural and the intrathecal approach with externalized or implanted catheters and infusion pumps. Clin J Pain 1998; 14:4-16.
114. Buchser E, Durrer A, Chedel D, Mustaki JP. Efficacy of intrathecal bupivacaine: How important is the flow rate? Pain Med 2004; 5:248-252.

115. Kumar K, Bodani V, Bishop S, Tracey S. Use of intrathecal bupivacaine in refractory chronic nonmalignant pain. Pain Med 2009; 10:819-828.

116. van Dongen RT, van Ee R, Crul BJ. Neurological impairment during long-term intrathecal infusion of bupivacaine in cancer patients: A sign of spinal cord compression. Pain 1997; 69:205-209.

117. Flack SH, Bernards CM. Cerebrospinal fluid and spinal cord distribution of hyperbaric bupivacaine and baclofen during slow intrathecal infusion in pigs. Anesthesiology 2010; 112:165-173.

118. Mercadante S, Arcuri E, Ferrera P, Villari $P$, Mangione S. Alternative treatments of breakthrough pain in patients receiving spinal analgesics for cancer pain. J Pain Symptom Manage 2005; 30:485-491.

119. Dahm P, Nitescu P, Appelgren L, Curelaru I. High thoracic/low cervical, longterm intrathecal (I.T.) infusion of bupivacaine alleviates "refractory" pain in patients with unstable angina pectoris. Report of 2 cases. Acta Anaesthesiol Scand 1998; 42:1010-1017.

120. Coffey RJ, Owens ML, Broste SK, Dubois MY, Ferrante FM, Schultz DM, Stearns LJ, Turner MS. Mortality associated with implantation and management of intrathecal opioid drug infusion systems to treat noncancer pain. Anesthesiology 2009; 111:881-891.

121. Coffey RJ, Owens ML, Broste SK, Dubois MY, Ferrante FM, Schultz DM, Stearns LJ, Turner MS. Medical practice perspective: Identification and mitigation of risk factors for mortality associated with intrathecal opioids for non-cancer pain Pain Med 2010; 11:1001-1009.

122. Allen JW, Horais KA, Tozier NA, Wegner K, Corbeil JA, Mattrey RF, Rossi SS, Yaksh TL. Time course and role of morphine dose and concentration in intrathecal granuloma formation in dogs: A combined magnetic resonance imaging and histopathology investigation. Anesthesiology 2006; 105:581-589.

123. Allen JW, Horais KA, Tozier NA, Yaksh TL. Opiate pharmacology of intrathecal granulomas. Anesthesiology 2006; 105:590-598.

124. Blount JP, Remley KB, Yue SK, Erickson DL. Intrathecal granuloma complicating chronic spinal infusion of morphine. Report of three cases. J Neurosurg 1996; 84:272-276. 
125. Cabbell KL, Taren JA, Sagher O. Spinal cord compression by catheter granulomas in high-dose intrathecal morphine therapy: Case report. Neurosurgery 1998; 42:1176-1180.

126. Coffey RJ, Burchiel K. Inflammatory mass lesions associated with intrathecal drug infusion catheters: Report and observations on 41 patients. Neurosurgery 2002; 50:78-86.

127. Miele VJ, Price KO, Bloomfield S, Hogg J, Bailes JE. A review of intrathecal morphine therapy related granulomas. Eur ] Pain 2006; 10:251-261.

128. Zacest AC, Carlson JD, Nemecek A, Burchiel KJ. Surgical management of spinal catheter granulomas: Operative nuances and review of the surgical literature. Neurosurgery 2009; 65:1161-1164.

129. Deer TR. A prospective analysis of intrathecal granuloma in chronic pain patients: A review of the literature and report of a surveillance study. Pain Physician 2004; 7:225-228.

130. Vandenbroucke JP, von Elm E, Altman DG, Gøtzsche PC, Mulrow CD, Pocock S), Poole C, Schlesselman JJ, Egger M; STROBE Initiative. Strengthening the Reporting of Observational Studies in Epidemiology (STROBE): Explanation and elaboration. Ann Intern Med 2007; 147:W163-W194.

131. von Elm E, Altman DG, Egger M, Pocock SJ, Gøtzsche PC, Vandenbroucke JP; STROBE Initiative. The Strengthening the Reporting of Observational Studies in Epidemiology (STROBE) Statement: Guidelines for reporting observational studies. Ann Intern Med 2007; 147:573-577.

132. Healy D. Randomized controlled trials: Evidence biased psychiatry. Alliance for Human Research Protection, 2002. www.ahrp.org/COI/healyo802.php

133. Glasziou P, Vandenbroucke JP, Chalmers I. Assessing the quality of research. BM] 2004; 328:39-41.

134. Black N. Why we need observational studies to evaluate the effectiveness of health care. BMJ 1996; 312:1215-1218.

135. Funai EF, Rosenbush EJ, Lee MJ, Del Priore G. Distribution of study designs in four major US journals of obstetrics and gynecology. Gynecol Obstet Invest 2001; 51:8-11.

136. Scales CD Jr., Norris RD, Peterson BL, Preminger GM, Dahm P. Clinical research and statistical methods in the urology literature. J Urol 2005; 174:13741379.
137. Greene T. Are observational studies "just as effective" as randomized clinical trials? Blood Purif 2000; 18:317-322.

138. Papanikolaou PN, Christidi GD, loannidis JP. Comparison of evidence on harms of medical interventions in randomized and nonrandomized studies. CMA] 2006; 174:635-641.

139. Guyatt G, Drummond R. Part 1. The basics: Using the medical literature. IA. Introduction: The philosophy of evidencebased medicine. In: Users' Guides to the Medical Literature: A Manual for EvidenceBased Clinical Practice. American Medical Association, Chicago, 2002, pp 3-12.

140. Hempel CG. Philosophy of Natural Science. Prentice-Hall, Englewood Cliffs, 1966.

141. Popper KR. The Logic of Scientific Discovery. Cambridge University Press, London, 1958.

142. Altman DG, Schulz KF, Moher D, Egger M, Davidoff F, Elbourne D, Gøtzsche PC, Lang T; CONSORT GROUP (Consolidated Standards of Reporting Trials). The revised CONSORT statement for reporting randomized trials: Explanation and elaboration. Ann Intern Med 2001; 134:663-694.

143. Piaggio G, Elbourne DR, Altman DG, Pocock SJ, Evans SJ; CONSORT Group. Reporting of noninferiority and equivalence randomized trials: An extension of the CONSORT statement. JAMA 2006; 295:1152-1160.

144. Deeks JJ, Dinnes J, D'Amico R, Sowden AJ, Sakarovitch C, Song F, Petticrew M, Altman DG; International Stroke Trial Collaborative Group; European Carotid Surgery Trial Collaborative Group. Evaluating non-randomised intervention studies. Health Technol Assess 2003; 7:1173 .

145. Pocock SJ, Collier TJ, Dandreo KJ, de Stavola BL, Goldman MB, Kalish LA, Kasten LE, McCormack VA. Issues in the reporting of epidemiological studies: A survey of recent practice. BMJ 2004; 329:883.

146. Juni P, Altman DG, Egger M. Systematic reviews in health care: Assessing the quality of controlled clinical trials. BM] 2001; 323:42-46.

147. Egger M, Schneider M, Davey Smith G. Spurious precision? Meta-analysis of observational studies. BMJ 1998; 316:140-144.

148. Stroup DF, Berlin JA, Morton SC, Olkin I, Williamson GD, Rennie D, Moher D, Becker BJ, Sipe TA, Thacker SB. Meta- analysis of observational studies in epidemiology: A proposal for reporting. Meta-analysis of Observational Studies in Epidemiology (MOOSE) group. JAMA 2000; 283:2008-2012.

149. Bero LA, Jadad AR. How consumers and policy makers can use systematic reviews for decision making. Ann Intern Med 1997; 127:37-42.

150. Berlin JA, Rennie D. Measuring the quality of trials. JAMA 1999; 282:10831085.

151. Blettner M, Sauerbrei W, Schlehofer B, Scheuchenpflug T, Friedenreich C. Traditional reviews, meta-analyses and pooled analyses in epidemiology. Int J Epidemiol 1999; 28:1-9.

152. Radford MJ, Foody JM. How do observational studies expand the evidence base for therapy? JAMA 2001; 286:1228-1230.

153. Lee W, Bindman J, Ford T, Glozier N, Moran P, Stewart R, Hotopf M. Bias in psychiatric case-control studies: Literature survey. $\mathrm{Br}$ J Psychiatry 2007; 190:204-209.

154. Tooth L, Ware R, Bain C, Purdie DM, Dobson A. Quality of reporting of observational longitudinal research. Am J Epidemiol 2005; 161:280-288.

155. Bogardus ST Jr., Concato J, Feinstein AR. Clinical epidemiological quality in molecular genetic research: The need for methodological standards. JAMA 1999; 281:1919-1926.

156. Verhalen RD. Guidelines for documentation of epidemiologic studies. Am J Epidemiol 1981; 114:609-613.

157. Rennie D. CONSORT revised-improving the reporting of randomized trials. JAMA 285:2006-2007.

158. Zwarenstein $M$, Treweek $S$, Gagnier J, Altman DG, Tunis S, Haynes B, Oxman AD, Moher D; CONSORT group; Pragmatic Trials in Healthcare (Practihc) group. Improving the reporting of pragmatic trials: An extension of the CONSORT statement. BM] 2008; 337:12231226.

159. Hartz A, Bentler S, Charlton M, Lanska D, Butani Y, Soomro GM, Benson K. Assessing observational studies of medical treatments. Emerg Themes Epidemiol 2005; 2:8.

160. Kane RL. Approaching the outcomes question. In: Kane RL (ed). Understanding Health Care Outcomes Research. Aspen Publications, Gaithersburg, 1997, pp 1-15.

161. Farquhar C, Marjoribanks J, Basser R, Hetrick S, Lethaby A. High dose chemotherapy and autologous bone marrow or stem cell transplantation versus 
conventional chemotherapy for women with metastatic breast cancer. Cochrane Database Syst Rev 2005; CDo03142.

162. Manchikanti L, Pampati V. Research designs in interventional pain management: Is randomization superior, desirable or essential? Pain Physician 2002; 5:275-284.

163. Carragee EJ, Hurwitz EL, Cheng I, Carroll LJ, Nordin M, Guzman J, Peloso P, Holm LW, Côté P, Hogg-Johnson S, van der Velde G, Cassidy JD, Haldeman S; Bone and Joint Decade 2000-2010 Task Force on Neck Pain and Its Associated Disorders. Treatment of neck pain: Injections and surgical interventions: Results of the Bone and Joint Decade
2000-2010 Task Force on Neck Pain and Its Associated Disorders. Spine (Phila Pa 1976) 2008; 33:S153-S169.

164. Benson K, Hartz AJ. A comparison of observational studies and randomized, controlled trials. N Engl J Med 2000; 342:1878-1886.

165. Hartz A, Benson K, Glaser J, Bentler S, Bhandari M. Assessing observational studies of spinal fusion and chemonucleolysis. Spine (Phila Pa 1976) 2003; 28:2268-2275.

166. Concato J, Shah N, Horwitz RI. Randomized, controlled trials, observational studies, and the hierarchy of research designs. N Engl J Med 2000; 342:18871892.
167. Shrier I, Boivin JF, Steele RJ, Platt RW, Furlan A, Kakuma R, Brophy J, Rossignol M. Should meta-analyses of interventions include observational studies in addition to randomized controlled trials? A critical examination of underlying principles. Am J Epidemiol 2007; 166:1203-1209.

168. Deer TR, Krames E, Levy RM, Hassenbusch SJ, 3rd, Prager JP. Practice choices and challenges in the current intrathecal therapy environment: An online survey. Pain Med 2009; 10:304-309. 